\title{
PARALLEL DOMAIN DECOMPOSITION STRATEGIES FOR STOCHASTIC ELLIPTIC EQUATIONS. PART A: LOCAL KARHUNEN-LOÈVE REPRESENTATIONS*
}

\author{
ANDRES A. CONTRERAS ${ }^{\dagger}$, PAUL MYCEK ${ }^{\dagger \ddagger}$, OLIVIER P. LE MAÎTRE ${ }^{\S}$, \\ FRANCESCO RIZZI ${ }^{\uparrow}$, BERT DEBUSSCHERE $\uparrow$, AND OMAR M. KNIO ${ }^{\dagger}$
}

\begin{abstract}
This work presents a method to efficiently determine the dominant Karhunen-Loève (KL) modes of a random process with known covariance function. The truncated KL expansion is one of the most common techniques for the approximation of random processes, primarily because it is an optimal representation, in the mean squared error sense, with respect to the number of random variables in the representation. However, finding the KL expansion involves solving integral problems, which tends to be computationally demanding. This work addresses this issue by means of a work-subdivision strategy based on a domain decomposition approach, enabling the efficient computation of a possibly large number of dominant KL modes. Specifically, the computational domain is partitioned into smaller nonoverlapping subdomains, over which independent local KL decompositions are performed to generate local bases which are subsequently used to discretize the global modes over the entire domain. The latter are determined by means of a Galerkin projection. The procedure leads to the resolution of a reduced Galerkin problem, whose size is not related to the dimension of the underlying discretization space but is actually determined by the desired accuracy and the number of subdomains. It can also be easily implemented in parallel. Extensive numerical tests are used to validate the methodology and assess its serial and parallel performance. The resulting expansion is exploited in Part B to accelerate the solution of the stochastic partial differential equations using a Monte Carlo approach.
\end{abstract}

Key words. domain decomposition, stochastic processes, Karhunen-Loève expansion

AMS subject classifications. 65M55, 60H15, 65M70, 31B10

DOI. $10.1137 / 17 \mathrm{M} 1132185$

1. Introduction. Stochastic partial differential equations (PDEs), and elliptic ones in particular, are increasingly being used to account for situations involving uncertain or incomplete knowledge of the simulated system and to perform, for example, inference tasks and sensitivity analyses. Computational approaches for the solution of Stochastic PDEs conceptually involve three essential steps: the modeling of the input uncertainty, the solution of the governing equations, and ultimately postprocessing the output to characterize the uncertainty. This paper (Part A) and its companion (Part B) focus on the two first steps, respectively. We discuss at present a domain decomposition strategy to approximate random fields using local reduced bases and local coordinates. These developments are motivated by the computational approach

*Submitted to the journal's Software and High-Performance Computing section May 30, 2017; accepted for publication (in revised form) April 30, 2018; published electronically July 17, 2018.

http://www.siam.org/journals/sisc/40-4/M113218.html

Funding: This work was supported by the US DOE, Office of Science, Office of Advanced Scientific Computing Research, under award DE-SC0010540. Sandia National Laboratories is a multimission laboratory managed and operated by National Technology and Engineering Solutions of Sandia, LLC, a wholly owned subsidiary of Honeywell International, Inc., for the US DOE's National Nuclear Security Administration under contract DE-NA-0003525. Support of the King Abdullah University of Science and Technology is also acknowledged.

${ }^{\dagger}$ Duke University, Durham, NC 27708 (andres.contreras@duke.edu).

${ }^{\ddagger}$ CERFACS, Toulouse, 31057 France (mycek@cerfacs.fr).

$\S$ CNRS, LIMSI, Université Paris Saclay, Orsay, France (olm@limsi.fr).

『Sandia National Laboratories, Livermore, CA 94550 (fnrizzi@sandia.gov, bjdebus@sandia.gov).

"King Abdullah University of Science and Technology, Thuwal, Saudi Arabia (omar.knio@kaust. edu.sa).

C520

Copyright $@$ by SIAM. Unauthorized reproduction of this article is prohibited. 
proposed in Part B, where the structure of local representations is exploited to accelerate the Monte Carlo sampling of the solution.

Modeling and approximating random processes are important tasks in many applications and in particular in uncertainty quantification problems. Focusing on random processes in spatial domains, it is often convenient to perform a discretization of the process in both stochastic and spatial dimensions for computational purposes. One way to achieve this is to rely on the representation of the process in terms of random coordinates in a spatial basis and proceed with the discretization of the spatial basis functions and random coordinates. An overview of several stochastic-discretization methods for random fields is provided in [21, 27, 26]. Among these methods, a common one in the area of computational stochastic mechanics is the Karhunen-Loève (KL) expansion $[17,12,11]$. A key advantage of the KL expansion is that it is optimal, with respect to the number of random variables involved in the representation, in the mean squared error sense. This is particularly attractive when using stochastic spectral methods, such as polynomial chaos expansions [5, 15], in view of analyzing the influence of the process on a model solution, that is, performing uncertainty quantification. Indeed, these functional representation methods exploit heavily the smoothness of the model solution with respect to the stochastic coordinates appearing in the KL decomposition. A challenge, however, is that finding the KL expansion of a stochastic process involves decomposing its covariance function. This leads to solving a Fredholm integral equation of the second kind for a kernel consisting of the twopoint covariance function. Decomposing such kernels is computationally demanding, because large spatial discretization grids are routinely considered when solving PDEs. A detailed description of Fredholm integral equations of the second kind is provided in [1], and a comparison of different methods that solve the KL expansion is found in [3].

For some particular covariance kernels, an analytical solution of the Fredholm integral equation is available (see, for instance, [5]). However, this is not typically the case, and numerical procedures are required for its spectral decomposition [7]. Different approaches have been devised in order to make more tractable the computation of the KL expansion. For instance, in [23] a wavelet-Galerkin approach is discussed, which provides localized support leading to sparse matrix equations that can be solved at a reduced cost. Another approach is presented in [25], which relies on fast multipole methods to speed up the computations. A more recent attempt at making the problem more tractable is provided in [3], but still finding the KL expansion remains a challenging problem, especially in situations where a large number of terms are needed or the physical space is multidimensional.

This paper proposes an efficient parallel method for the computation of KL expansions with a potentially large number of terms (broad spectrum). The method is based on a domain decomposition technique and we refer to it as the domain decomposition KL (DD-KL) method. The proposed strategy involves partitioning the computational domain into smaller nonoverlapping subdomains, over which local KL decomposition problems are solved to generate local bases. Our approach elucidates the exact correlation structure between sets of local coordinates associated to different subdomains and is composed of the following three main stages: (i) solving a local $K L$ expansion problem over each subdomain, (ii) using the dominant eigenfunctions from the local expansions to assemble a reduced eigenvalue problem, and (iii) solving the reduced eigenvalue problem to obtain the desired (global) KL expansion. Our representation is exploited in Part B to accelerate the solution of elliptic PDEs using a Monte Carlo procedure, specifically applying the local representations to expand 
the condensed stochastic problem of the domain decomposition formulation as local polynomial chaos expansions.

In addition to the immediate computational advantages of (i) solving small independent local problems and (ii) solving a low dimensional reduced global one, the method also allows us to efficiently distribute and parallelize most of the computations. Finally, as mentioned above, the method yields a representation in terms of independent stochastic coordinates that is convenient to perform uncertainty quantification tasks and sensitivity analyses, to characterize, for instance, the impact of the random process on a model solution. Indeed, the expansion in terms of independent random coordinates enables both sampling based approaches (e.g., Monte Carlo) and functional expansions (e.g., polynomial chaos and low rank approximations $[5,14,22,28])$. Furthermore, the global random coordinates can be related to small sets of local coordinates, over the subdomains, to reduce the computational complexity of the uncertainty quantification problem based on the KL approximation of the process.

The outline of the paper is as follows. In section 2, we recall the KL expansion and detail the proposed domain decomposition method. Also in this section, a truncation strategy for the DD-KL method is established in order to control the error in the resulting approximation. In section 3, numerical results are provided illustrating the effectiveness of the DD-KL method and the error control. Next, section 4 is dedicated to illustrating both the serial behavior of the approach and its parallel scalability and efficiency. Finally, in section 5, some concluding remarks are provided.

2. A domain decomposition method for KL expansions. In this section we introduce the proposed domain decomposition method to approximate the KL expansion of a stochastic process. We start by introducing several notations used throughout the paper. Consider a probability space $\mathcal{P}=\left(\Theta, \Sigma_{\Theta}, \mu_{\Theta}\right)$, where $\Theta$ is the set of events, $\Sigma_{\Theta}$ a sigma-algebra over $\Theta$, and $\mu_{\Theta}$ a probability measure. We denote by $\mathbb{E}[\cdot]$ the expectation operator,

$$
\mathbb{E}[U]=\int_{\Theta} U(\theta) d \mu_{\Theta}(\theta),
$$

and $L_{2}(\Theta)$ the corresponding space of second-order random variables, i.e., such that $\mathbb{E}\left[U^{2}\right]<+\infty$. At the deterministic level, we consider $\Omega$ a bounded subset of $\mathbb{R}^{n}$ (with $n=1,2$, or 3 ) and define $L_{2}(\Omega)$ the space of square integrable functionals $f: \boldsymbol{x} \in \Omega \mapsto f(\boldsymbol{x}) \in \mathbb{R}$. We shall denote by $\|\cdot\|_{\Omega}$ the norm in $L_{2}(\Omega)$ induced by the scalar product $\langle\cdot, \cdot\rangle_{\Omega}$ :

$$
\forall f \in L_{2}(\Omega), \quad\|f\|_{\Omega}^{2}=\langle f, f\rangle_{\Omega}=\int_{\Omega}|f(\boldsymbol{x})|^{2} d \boldsymbol{x} .
$$

Finally, we denote by $L_{2}(\Omega, \Theta)$ the space of real-valued second-order processes $U$ : $\Omega \times \Theta \rightarrow \mathbb{R}$ such that $U(\cdot, \theta) \in L_{2}(\Omega), U(\boldsymbol{x}, \cdot) \in L_{2}(\Theta)$ and

$$
\mathbb{E}\left[\|U(\cdot, \theta)\|_{\Omega}^{2}\right]<+\infty \Leftrightarrow U \in L_{2}(\Omega, \Theta) .
$$

2.1. The KL expansion. Let $U \in L_{2}(\Omega, \Theta)$ be a centered random process with known covariance $C: \Omega \times \Omega \rightarrow \mathbb{R}$ :

$$
C\left(\boldsymbol{x}, \boldsymbol{x}^{\prime}\right) \equiv \mathbb{E}\left[U(\boldsymbol{x}, \cdot) U\left(\boldsymbol{x}^{\prime}, \cdot\right)\right] .
$$

The truncated KL approximation, $U_{N}$, of a second-order stochastic process $U$, consists in an $N$-term expansion where each term is composed of the product of a deterministic 
function of $L_{2}(\Omega)$ with a random variable of $L_{2}(\Theta)$. The KL approximation $U_{N}$ is defined so as to minimize the representation error $U-U_{N}$ in the $L_{2}(\Omega, \Theta)$ sense. As covariance functions are symmetric and nonnegative, it can be shown that the sought $\mathrm{KL}$ expansion is given by

$$
U_{N}(\boldsymbol{x}, \theta) \equiv \sum_{\alpha=1}^{N} \sqrt{\lambda_{\alpha}} \eta_{\alpha}(\theta) \Phi_{\alpha}(\boldsymbol{x})
$$

where the $\lambda_{\alpha}$ 's and the $\Phi_{\alpha}$ 's are the (dominant) eigenvalues and associated (normalized) eigenfunctions of the covariance, i.e., the solution to the integral equation

$$
\int_{\Omega} C\left(\boldsymbol{x}, \boldsymbol{x}^{\prime}\right) \Phi\left(\boldsymbol{x}^{\prime}\right) d \boldsymbol{x}^{\prime}=\lambda \Phi(\boldsymbol{x}), \quad\langle\Phi, \Phi\rangle_{\Omega}=1 .
$$

The $\eta_{\alpha}(\theta)$ are the stochastic coordinates of $U$, having the property of being orthonormal:

$$
\mathbb{E}\left[\eta_{\alpha} \eta_{\beta}\right]= \begin{cases}1, & \alpha=\beta \\ 0 & \text { otherwise. }\end{cases}
$$

Owing to the structure of the covariance function, $C$, the eigenvalues are nonnegative and can be ordered in decreasing magnitude, leading to a natural energy criterion for the truncation of the expansion. Classically, $N$ is fixed so as to satisfy some error tolerance in the $L_{2}(\Omega, \Theta)$ norm. Specifically, one sets $N$ so the following inequality is satisfied for some prescribed error tolerance $0<\delta<1$ :

$$
\mathbb{E}\left[\left\|U-U_{N}\right\|_{\Omega}^{2}\right]=\mathbb{E}\left[\|U\|_{\Omega}^{2}\right]-\sum_{\alpha=1}^{N} \lambda_{\alpha} \leq \mathbb{E}\left[\|U\|_{\Omega}^{2}\right] \delta^{2} .
$$

Note that the process norm can be evaluated from the covariance function, $\mathbb{E}\left[\|U\|_{\Omega}^{2}\right]=$ $\int_{\Omega} C(\boldsymbol{x}, \boldsymbol{x}) d \boldsymbol{x}$, or through the whole KL spectrum: $\mathbb{E}\left[\|U\|_{\Omega}^{2}\right]=\sum_{\alpha} \lambda_{\alpha}$.

Galerkin approximation. In most cases, an analytical solution of (6) is not available, and we have to rely on a numerical method to approximate the eigenvalues and eigenfunctions. To this end, we choose a finite dimensional space $\mathcal{V}$, consisting of the linear span of a basis $\left\{v_{1}(\boldsymbol{x}), v_{2}(\boldsymbol{x}), \ldots, v_{\mathrm{Ne}}(\boldsymbol{x})\right\}$, with basis functions $v_{i} \in L_{2}(\Omega)$. Let $\Phi^{h}(\boldsymbol{x})=\sum_{k=1}^{\mathrm{Ne}} c_{k} v_{k}(\boldsymbol{x}) \in \mathcal{V}$ be the (finite dimensional) approximation and $\Phi(\boldsymbol{x})$. Using the approximate into (6) gives the residual

$$
r(\boldsymbol{x}) \equiv \lambda \Phi^{h}(\boldsymbol{x})-\int_{\Omega} C\left(\boldsymbol{x}, \boldsymbol{x}^{\prime}\right) \Phi^{h}\left(\boldsymbol{x}^{\prime}\right) d \boldsymbol{x}^{\prime}=\sum_{k=1}^{\mathrm{Ne}} c_{k}\left(\lambda v_{k}(\boldsymbol{x})-\int_{\Omega} C\left(\boldsymbol{x}, \boldsymbol{x}^{\prime}\right) v_{k}\left(\boldsymbol{x}^{\prime}\right) d \boldsymbol{x}^{\prime}\right) .
$$

In the Galerkin method, the vector of coefficients $\mathbf{c}=\left(c_{1}, \ldots, c_{\mathrm{Ne}}\right)^{T}$ is chosen by forcing $r(\boldsymbol{x})$ to be orthogonal to all functions in $\mathcal{V}$, i.e.,

$$
\langle r, u\rangle_{\Omega}=0 \quad \forall u \in \mathcal{V} .
$$

Substituting (9) into (10) and exploiting the structure of $\mathcal{V}$, the problem can be recast as a generalized eigenvalue problem:

$$
[K] \mathbf{c}=\lambda[M] \mathbf{c},
$$

Copyright $@$ by SIAM. Unauthorized reproduction of this article is prohibited. 
where $[K]$ and $[M]$ are nonnegative symmetric matrices of $\mathbb{R}^{\mathrm{Ne} \times \mathrm{Ne}}$ with entries

$$
[K]_{i j}=\int_{\Omega} \int_{\Omega} C\left(\boldsymbol{x}, \boldsymbol{x}^{\prime}\right) v_{i}\left(\boldsymbol{x}^{\prime}\right) v_{j}(\boldsymbol{x}) d \boldsymbol{x}^{\prime} d \boldsymbol{x}, \quad[M]_{i j}=\left\langle v_{i}, v_{j}\right\rangle_{\Omega} .
$$

The dimension Ne of the discrete generalized eigenvalue problem (11) depends on the dimension of the approximation space $\mathcal{V}$. If a finite element $(\mathrm{FE})$ method is used to discretize the problem, the dimension of the basis is equal to the number of degrees of freedom $\mathrm{Ne}$ of the $\mathrm{FE}$ space, which is determined by the number and type of elements used. Depending on the covariance structure, a very fine discretization (i.e., very large $\mathrm{Ne}$ ) might be required to accurately represent the eigenfunctions, and, as a result, solving the eigenvalue problem could become computationally intensive. This prevents the use of direct methods for most practical problems with large Ne. As one is generally not interested in the full KL decomposition of $U$ but in the $N$ dimensional dominant subspace, iterative methods (e.g., subspace iterations, Arnoldi, Lanczos; see, for instance, [6]) can be considered as an alternative for the resolution of (11). However, although for typical finite element discretizations the mass matrix $[M]$ is sparse, most covariance functions induce a full matrix $[K]$, with possibly prohibitive memory requirements for its storage. Matrix-free iterative methods and parallel implementation can be used to overcome memory limitations and accelerate computations, but the full character of the operator $[K]$ induces large computational cost and important communication burdens that can severely impact the efficiency of these approaches.

In the following subsection, we introduce an alternative strategy suitable to the parallel computation of the KL decomposition. It uses a domain decomposition approach to conveniently distribute the computational load among several processors and recast (11) in a reduced eigenvalue problem having a size dictated by the targeted error tolerance rather than by the dimension of the underlying discretization space, e.g., Ne.

2.2. Domain decomposition approach. As illustrated in Figure 1, our approach starts by partitioning the domain $\Omega$ into $D$ nonoverlapping subdomains:

$$
\bar{\Omega}=\overline{\bigcup_{d=1}^{D} \Omega_{d}}, \quad \Omega_{i} \cap \Omega_{j \neq i}=\emptyset .
$$

Next, for each subdomain $\Omega_{d}, d=1, \ldots, D$, we introduce the local eigenmodes $\tilde{\phi}_{\beta}^{(d)}: \Omega_{d} \rightarrow \mathbb{R}$, defined as the solutions of

$$
\int_{\Omega_{d}} C\left(\boldsymbol{x}, \boldsymbol{x}^{\prime}\right) \tilde{\phi}_{\beta}^{(d)}\left(\boldsymbol{x}^{\prime}\right) d \boldsymbol{x}^{\prime}=\lambda_{\beta}^{(d)} \tilde{\phi}_{\beta}^{(d)}(\boldsymbol{x}), \quad\left\|\tilde{\phi}_{\beta}^{(d)}\right\|_{\Omega_{d}}=1 .
$$

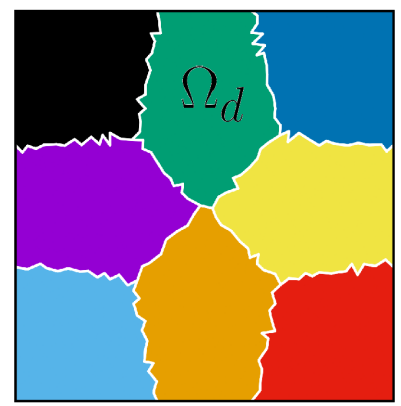

FIG. 1. Partitioning of a square domain $\Omega$ into $D=8$ nonoverlapping subdomains. 
In (14), we have denoted by $\|\cdot\|_{\Omega_{d}}$ the natural restriction of the norm in $L_{2}(\Omega)$ to the subdomain $\Omega_{d}$. It is seen that the $\tilde{\phi}_{\beta}^{(d)}$ are the eigenfunctions of the correlation $C\left(\boldsymbol{x}, \boldsymbol{x}^{\prime}\right)$ restricted to the $d$ th subdomain. These local eigenfuctions are extended to the global domain $\Omega$ by defining

$$
\forall \boldsymbol{x} \in \bar{\Omega}, \quad \phi_{\beta}^{(d)}(\boldsymbol{x})= \begin{cases}\tilde{\phi}_{\beta}^{(d)}(\boldsymbol{x}), & \boldsymbol{x} \in \Omega_{d}, \\ 0, & \boldsymbol{x} \notin \Omega_{d} .\end{cases}
$$

We observe that because the $\phi_{\beta}^{(d)}$ are orthonormal in $\Omega_{d}$, in light of (15), we have

$$
\left\langle\phi_{\beta}^{(d)}, \phi_{\beta^{\prime}}^{\left(d^{\prime}\right)}\right\rangle_{\Omega}= \begin{cases}1 & \text { if } d=d^{\prime} \text { and } \beta=\beta^{\prime}, \\ 0 & \text { otherwise. }\end{cases}
$$

For each subdomain $\Omega_{d}$ we retain the $m_{d}>0$ dominant eigenpairs according to the criterion discussed later in section 2.3. The $D$ sets of dominant eigenfunctions are collected to form an orthonormal reduced basis $\mathscr{B}$ of $L_{2}(\Omega)$ :

$$
\mathscr{B}=\bigcup_{d=1}^{D} \mathscr{B}_{d}, \quad \mathscr{B}_{d}=\left\{\phi_{\beta}^{(d)}, \beta=1, \ldots, m_{d}\right\} .
$$

We denote by $\mathcal{V}_{\mathscr{B}}$ the linear span of $\mathscr{B}$. We then seek an approximation $\hat{\Phi} \in \mathcal{V}_{\mathscr{B}}$ of the global modes solution of (6), that is,

$$
\Phi(\boldsymbol{x}) \approx \hat{\Phi}(\boldsymbol{x})=\sum_{d=1}^{D} \sum_{\beta=1}^{m_{d}} a_{\beta}^{(d)} \phi_{\beta}^{(d)}(\boldsymbol{x}) .
$$

Plugging the reduced basis approximation $\hat{\Phi}(\boldsymbol{x})$ of the global mode into the integral equation (6) and requiring the residual to be orthogonal to the reduced basis functions (i.e., applying a Galerkin method), leads to

$$
\forall \phi_{\beta}^{(d)} \in \mathscr{B}:\left\langle\int_{\Omega} C\left(\boldsymbol{x}, \boldsymbol{x}^{\prime}\right) \hat{\Phi}\left(\boldsymbol{x}^{\prime}\right) d \boldsymbol{x}^{\prime}, \phi_{\beta}^{(d)}\right\rangle_{\Omega}=\Lambda\left\langle\hat{\Phi}, \phi_{\beta}^{(d)}\right\rangle_{\Omega} .
$$

Using (18), this equation becomes, for all $\phi_{\beta}^{(d)} \in \mathscr{B}$,

$$
\begin{gathered}
\sum_{d^{\prime}=1}^{D} \sum_{\alpha=1}^{m_{d^{\prime}}} a_{\alpha}^{\left(d^{\prime}\right)}\left\langle\int_{\Omega} C\left(\boldsymbol{x}, \boldsymbol{x}^{\prime}\right) \phi_{\alpha}^{\left(d^{\prime}\right)}\left(\boldsymbol{x}^{\prime}\right) d \boldsymbol{x}^{\prime}, \phi_{\beta}^{(d)}\right\rangle_{\Omega} \\
=\Lambda \sum_{d^{\prime}=1}^{D} \sum_{\alpha=1}^{m_{d^{\prime}}} a_{\alpha}^{\left(d^{\prime}\right)}\left\langle\phi_{\alpha}^{\left(d^{\prime}\right)}, \phi_{\beta}^{(d)}\right\rangle_{\Omega}=\Lambda a_{\beta}^{(d)} .
\end{gathered}
$$

Denoting $\mathbf{a}^{(d)}=\left(a_{1}^{(d)}, \ldots, a_{m_{d}}^{(d)}\right)^{T}$ the vector of the local coordinates of $\hat{\Phi}$, and using the orthonormal character of the reduced basis, (20) can be recast as the following discrete eigenvalue problem:

$$
\left[\begin{array}{cccc}
{\left[\hat{K}_{11}\right]} & {\left[\hat{K}_{12}\right]} & \cdots & {\left[\hat{K}_{1 D}\right]} \\
{\left[\hat{K}_{21}\right]} & {\left[\hat{K}_{22}\right]} & \cdots & {\left[\hat{K}_{2 D}\right]} \\
\vdots & \vdots & \ddots & \vdots \\
{\left[\hat{K}_{D 1}\right]} & {\left[\hat{K}_{D 2}\right]} & \cdots & {\left[\hat{K}_{D D}\right]}
\end{array}\right]\left\{\begin{array}{c}
\mathbf{a}^{(1)} \\
\mathbf{a}^{(2)} \\
\vdots \\
\mathbf{a}^{(D)}
\end{array}\right\}=\Lambda\left\{\begin{array}{c}
\mathbf{a}^{(1)} \\
\mathbf{a}^{(2)} \\
\vdots \\
\mathbf{a}^{(D)}
\end{array}\right\}
$$


where the block matrices $\left[\hat{K}_{d, d^{\prime}}\right] \in \mathbb{R}^{m_{d} \times m_{d^{\prime}}}$ have for respective entries

$$
\left[\hat{K}_{d, d^{\prime}}\right]_{\alpha, \beta}=\left\langle\int_{\Omega} C\left(\boldsymbol{x}, \boldsymbol{x}^{\prime}\right) \phi_{\alpha}^{(d)}\left(\boldsymbol{x}^{\prime}\right) d \boldsymbol{x}^{\prime}, \phi_{\beta}^{\left(d^{\prime}\right)}\right\rangle_{\Omega}=\int_{\Omega_{d}} \int_{\Omega_{d^{\prime}}} C\left(\boldsymbol{x}, \boldsymbol{x}^{\prime}\right) \phi_{\alpha}^{(d)}(\boldsymbol{x}) \phi_{\beta}^{\left(d^{\prime}\right)}\left(\boldsymbol{x}^{\prime}\right) d \boldsymbol{x} d \boldsymbol{x}^{\prime}
$$

for $1 \leq \alpha \leq m_{d}, 1 \leq \beta \leq m_{d^{\prime}}$. We refer to (21) as the condensed eigenvalue problem. The dimension of this problem is

$$
n_{t}=\sum_{d=1}^{D} m_{d}=\operatorname{card} \mathscr{B} .
$$

It is easily shown that the matrix $[\hat{K}] \in \mathbb{R}^{n_{t} \times n_{t}}$ is symmetric and positive definite if the covariance function is such that for all $u \in L_{2}(\Omega)$,

$$
\|u\|_{\Omega}>0 \Rightarrow\left\langle u, \int_{\Omega} C(\cdot, \boldsymbol{x}) u(\boldsymbol{x}) d \boldsymbol{x}\right\rangle_{\Omega}>0 .
$$

This assumption is satisfied for most covariance functions, in particular for the whole Matérn class, and should not be restrictive in practice. Consequently, the $n_{t}$ eigenvalues $\Lambda_{\alpha}$ of $[\hat{K}]$ can be ordered with decreasing magnitude as

$$
\Lambda_{1} \geq \Lambda_{2} \geq \cdots \geq \Lambda_{n_{t}} \geq 0 .
$$

Then, for reasons explained in section 2.3, we can select the smallest $\hat{N}, 1 \leq \hat{N} \leq n_{t}$, such that for a prescribed relative error tolerance $0 \leq \delta \leq 1$ we have

$$
\sum_{\alpha=\hat{N}+1}^{n_{t}} \Lambda_{\alpha} \leq \frac{\delta^{2}}{2} \sum_{\alpha=1}^{n_{t}} \Lambda_{\alpha}
$$

The truncated approximation of $U$ is then given by

$$
U(\boldsymbol{x}, \theta) \approx \hat{U}_{\hat{N}}(\boldsymbol{x}, \theta) \equiv \sum_{\alpha=1}^{\hat{N}} \sqrt{\Lambda_{\alpha}} \hat{\eta}_{\alpha}(\theta) \hat{\Phi}_{\alpha}(\boldsymbol{x}),
$$

where

$$
\hat{\Phi}_{\alpha}(\boldsymbol{x})=\sum_{d=1}^{D} \sum_{\beta=1}^{m_{d}} a_{\alpha, \beta}^{(d)} \phi_{\beta}^{(d)}(\boldsymbol{x})
$$

is the eigenfunction corresponding to $\Lambda_{\alpha}$.

As illustrated in the examples section, $n_{t}$ is essentially fixed by the requested accuracy and not by the size of the discretization space. In fact, in our simulations, typical values for $n_{t}$ were small enough to permit the use of direct solvers for the solution of the reduced problem. However, any other type of solver, for instance, iterative ones, can be considered for the solution of (21). In the following, we refer to the approximation (27) as the $D D-K L$ expansion. Algorithm 1 provides a schematic of the main steps involved in the DD-KL approach. The algorithm highlights the two main steps that can be carried out in parallel, namely, the construction of the local bases $\mathscr{B}_{d}$ and the assembly of the matrix $[\hat{K}]$ of the condensed operator. The actual parallel implementation of the method is further discussed and tested in section 4 .

Remark. A numerical method is needed to solve the local problems and yield the local eigenpairs $(\lambda, \phi)$; in the present work we use FE methods to discretize the Galerkin weak form of (6). Even though we mentioned in section 2.1 that using this approach could become computationally intractable for large problems, using it to solve the local problems is now a viable option, owing to the much smaller size of 


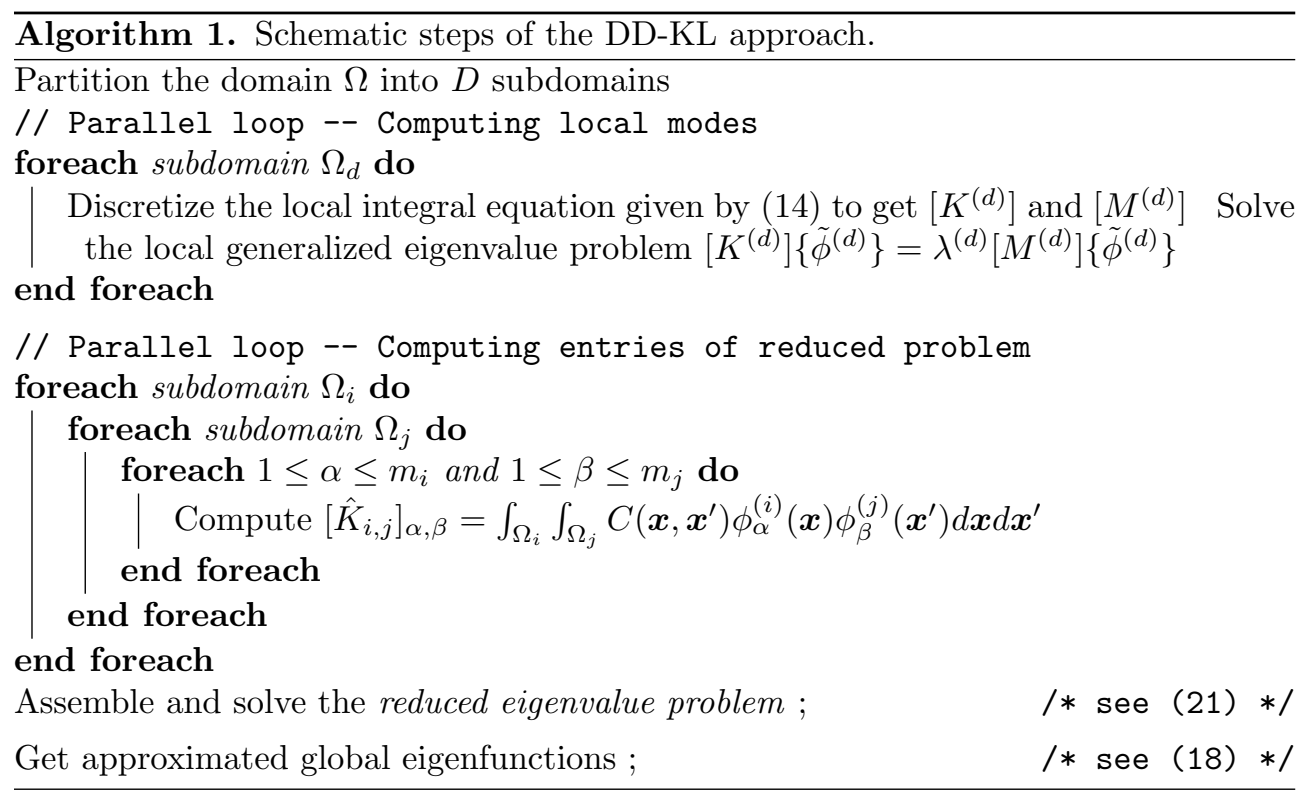

the local problems. Specifically, their number of degrees of freedom is approximately divided by $\mathrm{D}$ as compared to the global problem. Moreover, the local modes over distinct subdomains can be computed in parallel exploiting the independence of the local problems; this allows to effectively distribute the computational load among several processors.

Remark. The cost of solving the reduced eigenvalue problem is independent of the size of the discretization space used for the resolution of the local problem. Instead, it depends on the number of local modes retained in each subdomain, $m_{d}$, which add up to $n_{t}$; we expect $n_{t}=\mathcal{O}(N)$, which is in practice much less than Ne. The computational cost and complexity analysis of the method are discussed in section 4 on the basis of computational examples.

Remark. Even if the local modes $\phi_{\beta}^{(d)}(\boldsymbol{x})$ 's are all approximated using the same FE method ${ }^{1}$ over each subdomain $\Omega_{d}$, the condensed problem (21) does not ensure that the final approximation belongs to the FE space that would have been built over the whole domain $\Omega$. For instance, a typical situation corresponds to the case of continuous FE approximations over each subdomain $\Omega_{d}$ to compute the $\phi_{\beta}^{(d)}$ 's, so the $\hat{\Phi}_{\alpha}$ will generally be only piecewise continuous over the union of the $\Omega_{d}$ as indicated by (18), with jumps across the interfaces between subdomains. Such jumps are generally not a problem, but they can eventually be removed if needed at a postprocessing stage, e.g., by one of the averaging procedures routinely used in discontinuous Galerkin methods.

Remark. Substituting (18) into (27) we get the following alternative representation of the DD-KL expansion directly in terms of the local eigenmodes:

$$
\hat{U}_{\hat{N}}(\boldsymbol{x}, \theta)=\sum_{d=1}^{D}\left[\sum_{\beta=1}^{m_{d}} \sqrt{\lambda_{\beta}^{(d)}} \xi_{\beta}^{(d)}(\theta) \phi_{\beta}^{(d)}(\boldsymbol{x})\right], \quad \xi_{\beta}^{(d)}(\theta)=\sum_{\alpha=1}^{\hat{N}} \sqrt{\frac{\Lambda_{\alpha}}{\lambda_{\beta}^{(d)}}} a_{\alpha, \beta}^{(d)} \hat{\eta}_{\alpha}(\theta)
$$

\footnotetext{
${ }^{1}$ In fact, distinct subdomains could be treated using different discretization methods.
} 
where the $\left\{\xi_{\beta}^{(d)}(\theta), \beta=1, \ldots, m_{d}\right\}$ are called the local random variables. This expression highlights that for $\boldsymbol{x} \in \Omega_{d}$ the process $U(\boldsymbol{x}, \theta)$ can be approximated using an expansion that depends only on the local coordinates $\xi_{1 \leq \beta \leq m_{d}}^{(d)}$. In other words, even though the stochastic dimension of the truncated expansion is $n_{t}$, at the subdomain level we can characterize the process using a reduced stochastic dimension $m_{d}$. Equation (29) also shows that the local coordinates of a subdomain are, by construction, uncorrelated, but that the coordinates of distinct subdomains are generally correlated. Indeed, the correlation can be approximated as

$$
C\left(\boldsymbol{x} \in \Omega_{d}, \boldsymbol{x}^{\prime} \in \Omega_{d^{\prime}}\right) \approx \sum_{\beta=1}^{m_{d}} \sum_{\beta^{\prime}=1}^{m_{d^{\prime}}} \sqrt{\lambda_{\beta}^{(d)} \lambda_{\beta^{\prime}}^{\left(d^{\prime}\right)}} \mathbb{E}\left[\xi_{\beta}^{(d)} \xi_{\beta^{\prime}}^{\left(d^{\prime}\right)}\right] \phi_{\beta}^{(d)}(\boldsymbol{x}) \phi_{\beta}^{\left(d^{\prime}\right)}\left(\boldsymbol{x}^{\prime}\right) .
$$

Therefore, if the local coordinates of distinct subdomains were uncorrelated, i.e., if we had $\mathbb{E}\left[\xi_{\beta}^{(d)} \xi_{\beta^{\prime}}^{\left(d^{\prime}\right)}\right]=0$ for all pairs $\left(\beta, \beta^{\prime}\right)$ and $d \neq d^{\prime}$, then for $\boldsymbol{x}$ and $\boldsymbol{x}^{\prime}$ belonging to different subdomains $\left(d \neq d^{\prime}\right)$, we would necessarily have $C\left(\boldsymbol{x} \in \Omega_{d}, \boldsymbol{x}^{\prime} \in \Omega_{d^{\prime}}\right)=0$. This representation of the process in terms of local random variables is exploited in the sequel to this paper (Part B) to accelerate the solution of stochastic elliptic PDEs using a Monte Carlo approach.

2.3. Truncation strategy. In this section we address the selection of the parameters governing the proposed method. An obvious question concerns the selection of the number $D$ of subdomains and the actual partition of $\Omega$. In the example section 3.3, we numerically illustrate the robustness of the method with respect to the partitioning of $\Omega$ in $D$ subdomains and investigate the effects of varying $D$. Here, we focus on the selection of the number of local modes $m_{d}$ and the appropriate truncation of the final DD-KL expansion for a fixed partition of $\Omega$.

The method introduces two different sources of error in the approximation of $U$ by $\hat{U}_{\hat{N}}$. First, an error is introduced when representing the eigenfunctions of $U$ in the finite dimensional space $\mathcal{V}_{\mathscr{B}}$ built on the local bases; see (18). Hereafter, we shall denote by $U_{\mathscr{B}}$ the projection of $U$ on $\mathcal{V}_{\mathscr{B}}$ :

$$
U_{\mathscr{B}}(\boldsymbol{x}, \theta)=\sum_{d=1}^{D} \sum_{\alpha=1}^{m_{d}} \sqrt{\lambda_{\alpha}^{(d)}} \eta_{\alpha}^{(d)}(\theta) \phi_{\alpha}^{(d)}(\boldsymbol{x}) .
$$

Second, the projected process $U_{\mathscr{B}}$ is further reduced, through the resolution of the reduced problem, to yield the final approximation $\hat{U}_{\hat{N}}$.

Because $U-U_{\mathscr{B}}$ is orthogonal to $U_{\mathscr{B}}-\hat{U}_{\hat{N}}$, the squared norm of the error $U-\hat{U}_{\hat{N}}$ can actually be broken down into two independent parts as follows:

$$
\mathbb{E}\left[\left\|U-\hat{U}_{\hat{N}}\right\|_{\Omega}^{2}\right]=\mathbb{E}\left[\left\|U-U_{\mathscr{B}}\right\|_{\Omega}^{2}\right]+\mathbb{E}\left[\left\|U_{\mathscr{B}}-\hat{U}_{\hat{N}}\right\|_{\Omega}^{2}\right]
$$

See Appendix A for the derivation. The first term is obtained by adding up the local contributions over the subdomains, which, by construction of the local modes, are given by

$$
\epsilon_{d}^{2} \equiv \mathbb{E}\left[\left\|U-U_{\mathscr{B}}\right\|_{\Omega_{d}}^{2}\right]=\mathbb{E}\left[\|U\|_{\Omega_{d}}^{2}\right]-\sum_{\alpha=1}^{m_{d}} \lambda_{\alpha}^{(d)} \quad \forall d=1, \ldots, D
$$


Then, gathering the local contributions, we end up with

$$
\epsilon_{\mathscr{B}}^{2} \equiv \mathbb{E}\left[\left\|U-U_{\mathscr{B}}\right\|_{\Omega}^{2}\right]=\sum_{d=1}^{D} \epsilon_{d}^{2}=\mathbb{E}\left[\|U\|_{\Omega}^{2}\right]-\sum_{d=1}^{D} \sum_{\alpha=1}^{m_{d}} \lambda_{\alpha}^{(d)} .
$$

Finally, similarly to the classical KL truncation error, the second error contribution is

$$
\epsilon_{\mathscr{B} \hat{N}}^{2} \equiv \mathbb{E}\left[\left\|U_{\mathscr{B}}-\hat{U}_{\hat{N}}\right\|_{\Omega}^{2}\right]=\mathbb{E}\left[\left\|U_{\mathscr{B}}\right\|_{\Omega}^{2}\right]-\sum_{\alpha=1}^{\hat{N}} \Lambda_{\alpha} .
$$

Since $\mathbb{E}\left[\left\|U_{\mathscr{B}}\right\|_{\Omega}^{2}\right] \leq \mathbb{E}\left[\|U\|_{\Omega}^{2}\right]$, the overall error can be estimated from

$$
\mathbb{E}\left[\left\|U-\hat{U}_{\hat{N}}\right\|_{\Omega}^{2}\right]=\epsilon_{\mathscr{B}}^{2}+\epsilon_{\mathscr{B} \hat{N}}^{2} \leq 2 \mathbb{E}\left[\|U\|_{\Omega}^{2}\right]-\sum_{d=1}^{D} \sum_{\alpha=1}^{m_{d}} \lambda_{\alpha}^{(d)}-\sum_{\alpha=1}^{\hat{N}} \Lambda_{\alpha} .
$$

This expression shows that to reduce the error, one needs to jointly increase the size of the local basis over all the subdomains and increase $\hat{N}$. Clearly, this suggests the existence of an optimal (in terms of computational efficiency) set of values for $m_{d}$ and $\hat{N}$. In this work, in order to achieve an overall relative error $0 \leq \delta \leq 1$, we simply enforce the following error levels. First, regarding the local errors, we require simply that the $m_{d}$ 's are selected to ensure, for each $d$,

$$
\epsilon_{d}^{2}=\mathbb{E}\left[\|U\|_{\Omega_{d}}^{2}\right]-\sum_{\alpha=1}^{m_{d}} \lambda_{\alpha}^{(d)} \leq \mathbb{E}\left[\|U\|_{\Omega_{d}}^{2}\right] \frac{\delta^{2}}{2}
$$

such that $\epsilon_{\mathscr{B}}^{2} \leq \mathbb{E}\left[\|U\|_{\Omega}^{2}\right] \delta^{2} / 2$. Then, $\hat{N}$ is selected so that (26) holds, which ensures that

$$
\mathbb{E}\left[\left\|U-\hat{U}_{\hat{N}}\right\|_{\Omega}^{2}\right] \leq \delta^{2} \mathbb{E}\left[\|U\|_{\Omega}^{2}\right]
$$

Remark. We observe that the selection of the $m_{d}$ 's in (36) balances the local relative (squared) error over the subdomains; this could be further improved by collecting the local eigenvalues for all the subdomains and selecting the $n_{t}$ dominant ones that ensure $\epsilon_{\mathscr{B}}^{2} \leq \delta^{2} / 2$. However, numerical experiments have shown that the local selection based on (36) is quite satisfactory, and in addition, it maintains a strict independence of the local bases construction.

3. Numerical examples. In this section we describe the test problem used to validate the domain decomposition approach (section 3.1) and present several numerical results that demonstrate the convergence and the error control (section 3.2) of the method. Finally, we provide in section 3.3 a brief analysis of the impact of the domain partitioning on the behavior of the method.

3.1. Test problem. The method is applied to the decomposition of a secondorder stochastic process $U(\boldsymbol{x}, \theta)$ defined over the two-dimensional unit square $\Omega=$ $[0,1]^{2}$. For the covariance of the process, we assume the classical stationary squared exponential structure, with characteristic correlation length $L$ and unit variance:

$$
C\left(\boldsymbol{x}, \boldsymbol{x}^{\prime}\right)=\exp \left(-\left\|\boldsymbol{x}-\boldsymbol{x}^{\prime}\right\|_{2}^{2} / L^{2}\right) \text {. }
$$


We stress that the proposed method is not limited to this specific covariance structure and domain shape. For the spatial discretization of the KL modes, we use piecewise constant FE discretization unless otherwise indicated. This low-order FE approximation space is chosen because of the application to stochastic elliptic PDEs we have in mind; in these applications, the stochastic coefficient field modeled by KL expansion is often considered as constant over the finite elements [4]. In any case, we stress that our method perfectly accommodates higher-order FE methods. The spatial mesh consists of a conforming triangulation of $\Omega$ into a set $\Sigma$ of Ne finite elements. In addition, the results presented in the following use sufficiently many finite elements to allow for the accurate approximation of the dominant eigenmodes. The set of elements is partitioned into $D$ subsets forming connected nonoverlapping subdomains $\Omega_{d}$ for $d=1, \ldots, D$. For the partitioner, we rely on a $k$-means geometrical clustering algorithm $[18,16,19]$. Nonetheless, the numerical behavior of the method does not depend on the particular approach used to generate the subdomains, provided it produces geometrically similar subdomains.

The correlation length $L$ has a marked effect on the spectral decay of the KL expansion. Figure 2 shows the magnitude of the ordered eigenvalues of $C\left(\boldsymbol{x}, \boldsymbol{x}^{\prime}\right)$. From the figure, we can appreciate the slower decay rate of $\lambda_{k}$ for smaller values of $L$. In fact, for the smallest correlation lengths tested, the leading eigenvalues are seen to be essentially equal with an asymptotic decay that is increasingly delayed as $L$ decreases. The slower the decay rate, the larger the number of terms required in the KL expansion to achieve a desired accuracy. For instance, setting $\delta^{2}=10^{-3}$ in (8), we get $N=3, N=8, N=259$, and $N=22,356$ for $L=10, L=1, L=0.1$, and $L=0.01$, respectively.

The DD-KL approach exploits the fact that the convergence behavior of the KL expansion, for fixed covariance structure, is governed by the magnitude of $L$ relative to the characteristic length of the domain. Indeed, decreasing (resp. increasing) the characteristic extent of the domain has a similar effect as increasing (resp. decreasing) the correlation length. In the DD-KL approach, increasing the number of subdomains allows us to reduce the extent of the subdomains, leading to an apparent larger $L$ and a faster spectral decay for the local expansions. This is illustrated in Figure 3, where the spectra of local expansions are shown for different values of the number

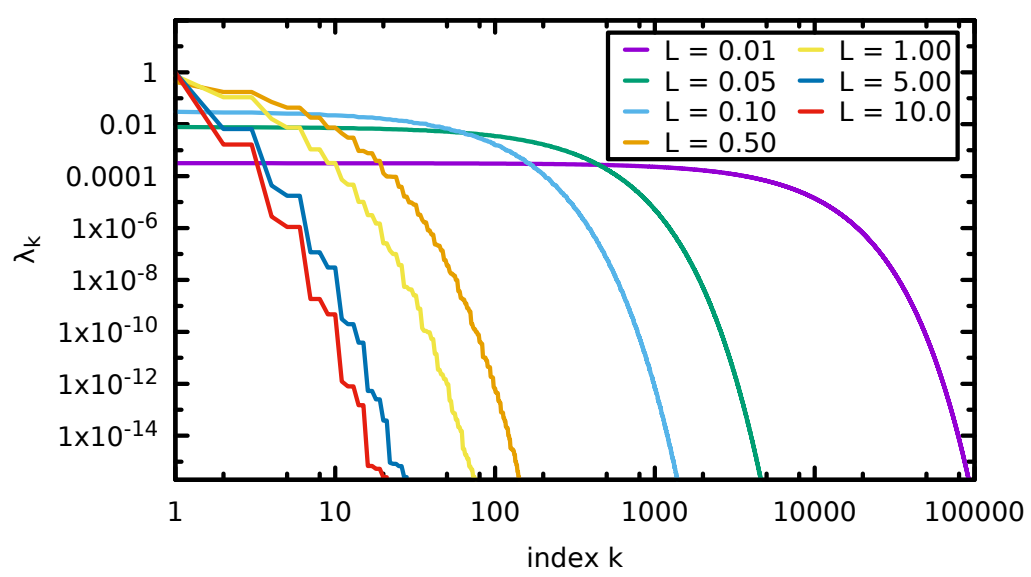

FIG. 2. Spectra of the global decomposition for the squared exponential covariance in (38) with different values of the correlation length $L$ as indicated. 


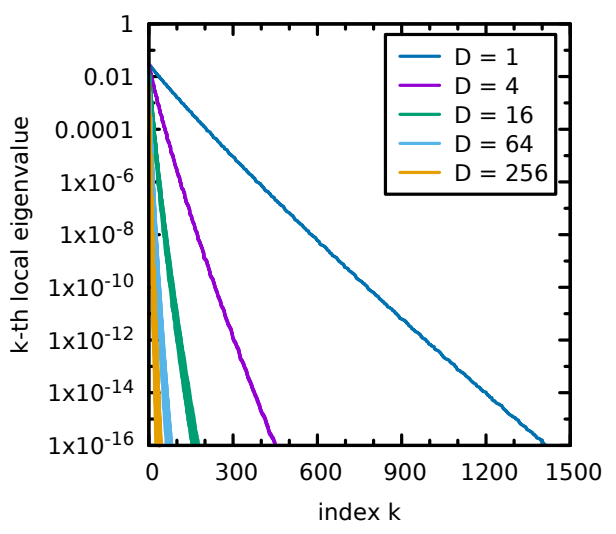

(a)

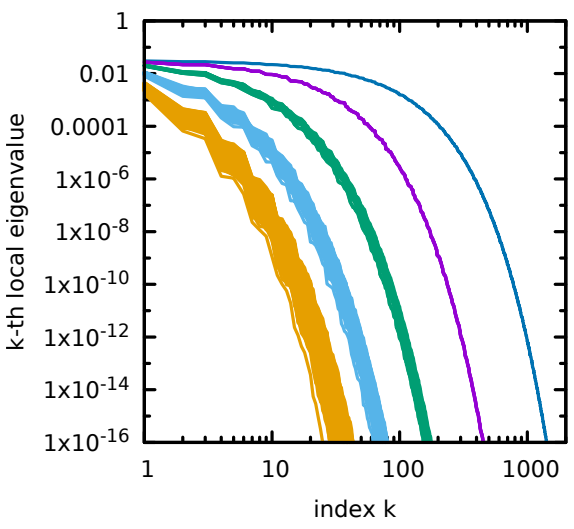

(b)

FIG. 3. Spectra of local decompositions for the squared Gaussian covariance (38), with $L=0.1$ and different $D$ as indicated. The plot in (a) uses a linear scale for the $x$-axis, whereas the plot in (b) uses a logarithmic scale.

of subdomains. Note that the figure reports the spectra for all the $D$ subdomains, so there are $D$ spectra plotted when $\Omega$ is partitioned into $D$ subdomains. Here, we observe that for a given number $D$ of subdomains, the local subdomains all have roughly the same extent so the local expansions have a similar decay. Moreover, it is seen that, as expected, the local expansions have spectra which decay faster as $D$ increases.

The variability of the spectra among the $D$ sudomains can be appreciated from Figure $3(\mathrm{~b})$. This variability is principally due to the partitioning procedure that generates nonidentical subdomains, with slightly variable apparent $L$ as a result.

3.2. Convergence analysis. We start by demonstrating that the proposed method converges towards the direct decomposition estimates of the dominant or truncated decomposition of $U$. To this end, we select arbitrarily truncation levels $N>0$ and check that the DD-KL solution $\hat{U}_{N}$ converges to the truncated direct solution $U_{N}$ (i.e., the solution computed without the domain decomposition) as the error tolerance is lowered. In fact, the comparison and convergence analyses are conducted using the spatially discretized solutions over the same finite element mesh. The convergence of (discrete) $\hat{U}_{N}$ toward the dominant approximation $U_{N}$ can be important in the context of stochastic elliptic equations, where recovering $U_{N}$ may be needed for sensitivity analysis and comparison purposes.

We compute $U_{N}$ solving (6) discretized over the finite element mesh, as described in section 2.1. The same finite element mesh with $\mathrm{Ne}=40,802$ elements and piecewise constant approximation is used for both decompositions. In this case, $\hat{U}_{N}$ and $U_{N}$ have the same dimensionality, and the differences are only due to the approximation of $\hat{U}_{N}$ in the reduced basis of local modes. To measure these differences, we define the following error metrics. First, we consider error in the spectra, computing the normalized $\ell_{1}$-distance between the $N$ dominant eigenvalues of the DD-KL solution $\left(\Lambda_{k}\right)$ and of the direct computation $\left(\lambda_{k}\right)$ :

$$
\epsilon_{\mathrm{spec}}=\frac{\sum_{k=1}^{N}\left|\lambda_{k}-\Lambda_{k}\right|}{\sum_{k=1}^{N}\left|\lambda_{k}\right|} .
$$


In addition to the error in spectra, $\epsilon_{\text {spec }}$, we quantify the distance between the $N$ dimensional subspaces of the direct and DD-KL eigen bases. Different error measures can be thought to characterize the distance between subspaces; here we simply rely on the expected squared $L_{2}$-norm of the projection error in the linear span of the eigenvectors $\hat{\Phi}_{\alpha}(\boldsymbol{x})$ of the DD-KL approximation. For a generic second-order process $V$, the (relative) subspace error measure $\epsilon_{\mathrm{sub}}^{2}(V)$ is defined as follows:

$$
\epsilon_{\text {sub }}^{2}(V)=\frac{\mathbb{E}\left[\left\|V(\boldsymbol{x}, \theta)-\sum_{\alpha=1}^{N}\left\langle V(\boldsymbol{x}, \theta), \hat{\Phi}_{\alpha}(\boldsymbol{x})\right\rangle_{\Omega} \hat{\Phi}_{\alpha}(\boldsymbol{x})\right\|_{\Omega}^{2}\right]}{\mathbb{E}\left[\|V(\boldsymbol{x}, \theta)\|_{\Omega}^{2}\right]} .
$$

Note that $\epsilon_{\text {sub }}^{2}\left(U_{N}\right)$ is therefore the spatially discretized version of the normalized projection error $\epsilon_{\mathscr{B}}$ in (33) for the truncated process $U_{N}$. In order to compute the expectation in the previous error definition, the process $U$ is assumed to be Gaussian in what follows.

For $N$ and $D$ fixed, we expect the difference between $U_{N}$ and $\hat{U}_{N}$ to decrease as richer and richer local bases are considered. Recalling that the dimension of the local basis for subdomain $\Omega_{d}$ is $m_{d}$, we expect $\hat{U}_{N} \rightarrow U_{N}$, in the sense of the metrics defined above, when $m_{d}$ increases for all the subdomains. As explained before, the local basis dimension $m_{d}$ yields a local expansion error $\epsilon_{d}$ given by (32). In the following, we choose a positive tolerance parameter $\delta^{*}$ to control the error in the local expansions, and we select the smallest $m_{d} \in \mathbb{N}_{+}$satisfying

$$
\epsilon_{d}^{2} \leq \mathbb{E}\left[\|U\|_{\Omega_{d}}^{2}\right] \times \delta^{*} \quad \forall d=1, \ldots, D .
$$

Figures 4 and 5 report the errors $\epsilon_{\text {spec }}$ and $\epsilon_{\text {sub }}^{2}$, respectively, as a function of the tolerance parameter $\delta^{*}$. The two figures show that one can improve the accuracy of the KL-DD expansion and approximate better and better $U_{N}$ by lowering the tolerance $\delta^{*}$, that is, by increasing the size of the local bases and accordingly enriching the reduced space. In the figures, a dashed line with unit slope is shown to serve as a visual reference corresponding to $\epsilon_{\mathrm{spec}}=\delta^{*}$ and $\epsilon_{\mathrm{sub}}^{2}=\delta^{*}$, respectively. We also note that as larger $N$ are considered, $\delta^{*}$ has to decrease consistently, because increasing $N$

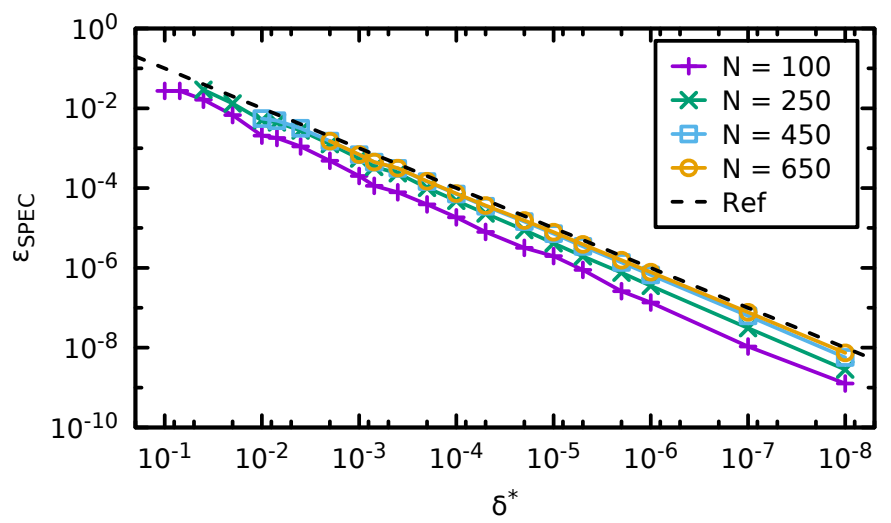

FIG. 4. Relative spectra error $\epsilon_{\text {spec, }}$, between $U_{N}$ and $\hat{U}_{N}$, as a function of the local tolerance $\delta^{*}$ (see (41)) for different subspace dimensions $N$ as indicated. Computations use $D=80$ and $L=0.1$. The dashed line has unit slope. 


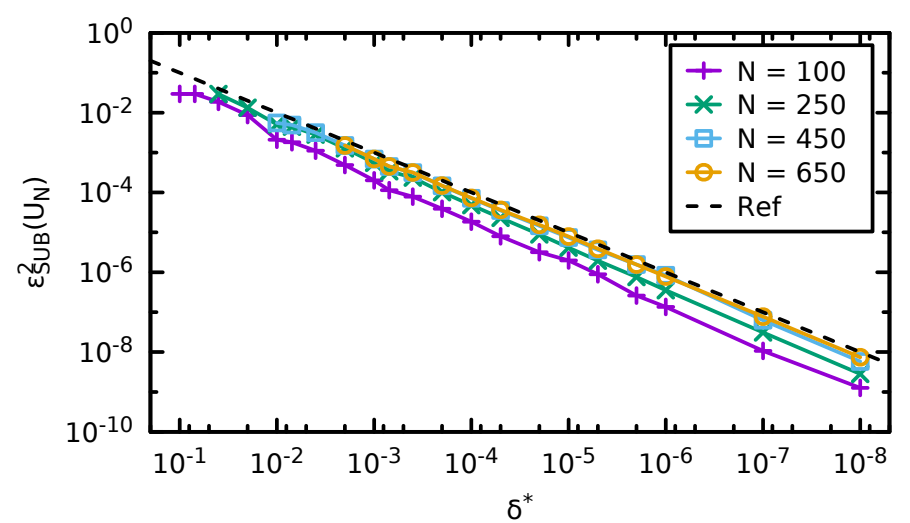

FIG. 5. Subspace error $\epsilon_{\text {sub }}^{2}\left(U_{N}\right)$ in (40) as a function of the local tolerance $\delta^{*}$ (see (41)) for different subspace dimensions $N$ as indicated. Computations use $D=80$ and $L=0.1$. The dashed line has unit slope.

requires $n_{t}=\sum_{d} m_{d} \geq N$; a minimal tolerance $\delta^{*}$ is therefore required to induce a reduced basis $\mathscr{B}$ large enough ( $\operatorname{card} \mathscr{B}>N$ ) when selecting the $m_{d}$ through (41). This explains the different ranges for $\delta^{*}$ considered in the plot, depending on $N$. Another interesting observation from Figures 4 and 5 is that the two errors decay proportionally with the local tolerance $\delta^{*}$. This trend demonstrates that the local accuracy fixes the global precision on $U_{N}$ for a fixed $N$ and $\delta^{*}$ small enough. Conversely, for a fixed and small enough $\delta^{*}$, the relative error $\epsilon_{\text {sub }}^{2}$ increases as we demand to approximate more and more modes (increasing $N$ ) but always remains less than $\delta^{*}$. Note that the error is always below the dashed line, which illustrates that we can effectively bound the error in the approximation to be less than any desired value $\delta^{*}$.

Now that we have established that the truncated DD-KL solution $\hat{U}_{N}$ converges to the truncated process $U_{N}$ when the tolerance on the local error is lowered, we proceed to investigate the error between $\hat{U}_{N}$ and the nontruncated process $U$. Strictly speaking, we actually look at the differences between the spatially discretized versions of $U$ and $\hat{U}_{N}$. Also, to compute efficiently the subspace error $\epsilon_{\mathrm{sub}}^{2}(U)$ in (40), we need either to directly sample realizations of $U$ or to know its (untruncated) KL expansion. Here, however, we use as a proxy for $U$ a truncated KL expansion of $U$, obtained with the direct method, with a very large number of modes, typically several times larger than the requested $N$. In Figure 6 , the evolution of $\epsilon_{\mathrm{sub}}^{2}(U)$ is shown as a function of the local tolerance $\delta^{*}$ for different values of $N$. These curves must be compared with the errors with respect to $U_{N}$ plotted in Figure 5. Contrary to the error with respect to $U_{N}$, the error with respect to $U$ is seen to level off and not to continue to decay when the tolerance $\delta^{*}$ is lowered. This behavior reflects the decomposition of the error in (31): when the projection error $\epsilon_{\mathscr{B}}$ is made smaller and smaller, lowering $\delta^{*}$, the truncation error $\epsilon_{\mathscr{B} \hat{N}}$ becomes dominant such that $U-\hat{U}_{N}$ cannot be reduced further but by increasing $N$. It confirms that increasing the dimension of the local bases beyond a certain threshold does not provide any appreciable advantages. On the contrary, it requires determining more local modes and solving a larger reduced problem with higher numerical cost as a result. The plots also verify the appropriateness of the strategy described in section 2.3 , since we can observe that for a given $N$ the relative subspace error stagnates after $\delta^{*} \approx \epsilon_{\mathrm{sub}}^{2}(U)$.

So far, we have kept the number of subdomains constant in analyzing the error in the DD-KL solution. We now show that the proposed error-control approach is not 


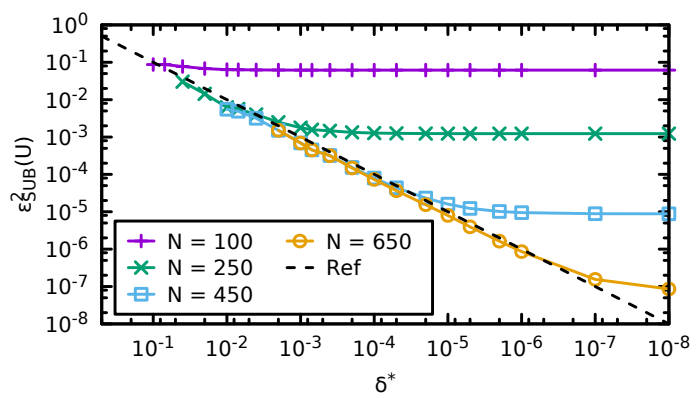

FIG. 6. Projection error $\epsilon_{\text {sub }}^{2}(U)$ with respect to the untruncated process, as a function of tolerance $\delta^{*}$ defining the local truncation through (41). Computations are conducted for $L=0.1$ using $D=80$ subdomains and different values of $N$ as indicated. The dashed black line is a reference of unit slope.

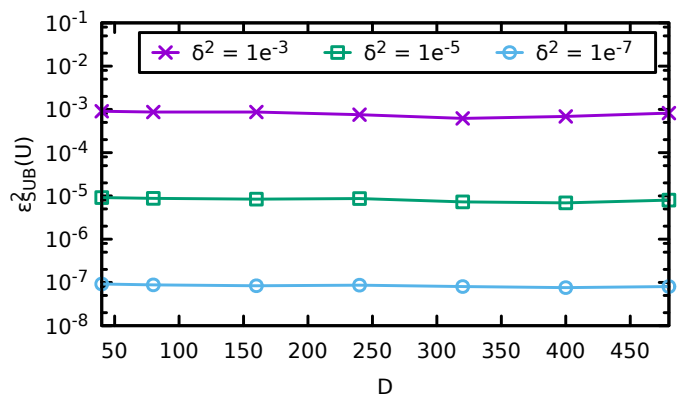

FIG. 7. Projection error $\epsilon_{\text {sub }}^{2}(U)$ as a function of the number of subdomains $D$ and for different targeted relative accuracy $\delta$ as indicated.

compromised when we increase the number of subdomains $D$ used to partition $\Omega$. In the following numerical test, we fix a target relative error $\delta$ and select the local and global truncation level using (26) and (36) to balance the projection $\left(\epsilon_{\mathscr{B}}^{2} \leq \frac{\delta^{2}}{2}\right)$ and truncation $\left(\epsilon_{\mathscr{B} \hat{N}}^{2} \leq \frac{\delta^{2}}{2}\right)$ errors. The DD-KL solution is then computed for different numbers $D$ of subdomains, and we report in Figure 7 the resulting relative projection error $\epsilon_{\mathrm{sub}}^{2}(U)$. The curves demonstrate that a relative error less than $\delta$ is consistently achieved irrespective of the number of subdomains considered.

The numerical experiments on the convergence of the DD-KL method were repeated using continuous piecewise quadratic FE discretizations $\left(P_{2}\right)$. We obtained results that are similar to the piecewise constant case, and, therefore, they are not reported here. Note, however, that the discretized DD-KL and direct solutions live in different finite dimensional space for the quadratic approximation, as the direct solution is continuous over the whole domain $\Omega$, when the DD-KL solution is only continuous over the subdomains $\Omega_{d}$, with possibly discontinuities across the boundaries between subdomains. Our numerical tests indicates that the DD-KL approach remains convergent for $\mathrm{FE}$ discretization with order greater than 0 and is not compromised as $D$ increases. Of course, changing the order of the FE method affects the error in the computed eigenmodes of the local problems, such that a coarser mesh with a lower number of degrees of freedom Ne could be considered for the $P_{2} \mathrm{FE}$ discretization, compared to the $P_{1}$ or $P_{0}$ discretizations, while maintaining a comparable accuracy in the final approximations. The present work is not concerned with the 
question of determining the optimal $\mathrm{FE}$ order and mesh size to achieve a prescribed accuracy at a minimal computational cost. This question is left as a subject of future studies.

3.3. Effect of domain partitioning. We have just seen that changing the number of subdomains does not affect the accuracy of the final DD-KL expansion, provided that the truncations of the local and final expansions are properly set. However, the number of subdomains is obviously expected to affect the computational cost, as the DD-KL method was motivated by the complexity reduction in the first place. The analysis of the computational efficiency of DD-KL method is delayed to the next section, where a parallel implementation is presented. In this section, we focus on the behavior of the local and reduced bases when $D$ varies and depending on the type of partioning of $\Omega$ considered.

We first assume that $\Omega$ is partitioned into $D$ subdomains having similar size. Following the discussion above, the change in apparent $L$ is proportional to $D^{1 / n}$ for $n$ spatial dimensions. In addition to the reduction of the dimensionality of the discretized local problems, we also expect the size of the local bases to decrease for larger $D$. However, the spectra shown in Figure 3(a) indicate that the reduction in the average (over the $D$ subdomains) number of local modes, $\bar{m}$, becomes marginally insignificant as $D$ increases. In any case, we have the lower bound $m_{d} \geq 1$, indicating that the behavior of the method for very large $D$ (compared to $N$ ) can be problematic. To clarify this point, we report in Table 1 the evolution of the reduced problem size $\left(n_{t}\right)$, average local basis dimension $(\bar{m})$, and standard deviation of $m_{d}$ (denoted $\sigma_{m_{d}}$ ), when increasing the numbers of subdomains. The results reported correspond to the previous example, with a target accuracy $\delta^{2}=2 \times 10^{-3}$. We observe that as $D$ increases, the dimension $n_{t}$ of the reduced problem increases. In fact, it is expected that asymptotically we would have $n_{t}=\mathcal{O}(\mathrm{Ne})$, where $\mathrm{Ne}$ is the number of degrees of freedom. This claim is supported by the evolutions of the averaged local basis dimension $\bar{m}$ which is seen to initially drop quickly and then continues to drop at a lower rate. For the range of $D$ investigated, the smallest value we reached was $\bar{m}=3$, but if $D$ continues to increase we will eventually converge to $\bar{m}=1$. Similarly, the standard deviation of the local basis dimension $m_{d}$ is reported to decay monotonically to zero. Since for a fixed target accuracy $\delta$ we have roughly fixed number of term $N$ in the expansion, the evolution of $n_{t}$ with $D$ indicates the existence of an optimal number of subdomains, balancing the complexity reduction in the local problems with the progressive increase in the size $n_{t}$ of the reduced problem. Determining this optimum is not obvious as it depends on implementation (e.g., how many local problems can be solved in parallel) and on the numerical method used to solve the reduced problem. Regardless of these considerations, we remark that, over the range of values for $D$

TABLE 1

Progression of $n_{t}$ for different values of $D$ with $\delta^{2}=2 \times 10^{-3}$ and $L=0.1$.

\begin{tabular}{ccc}
\hline $\mathrm{D}$ & $n_{t}$ & $\bar{m} \pm \sigma_{m_{d}}$ \\
\hline 20 & 431 & $21.55 \pm 1.43$ \\
40 & 542 & $13.55 \pm 0.59$ \\
80 & 741 & $9.26 \pm 0.56$ \\
160 & 983 & $6.14 \pm 0.35$ \\
320 & 1,682 & $5.26 \pm 0.44$ \\
640 & 2,306 & $3.60 \pm 0.53$ \\
1,280 & 3,840 & $3.00 \pm 0.00$ \\
\hline
\end{tabular}

Copyright $@$ by SIAM. Unauthorized reproduction of this article is prohibited. 


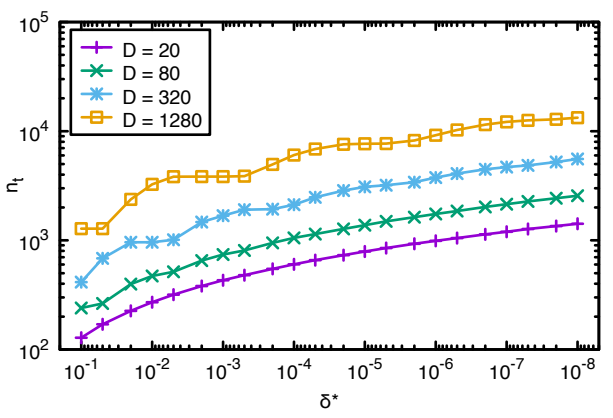

FIG. 8. Dimension $n_{t}$ of the reduced problem with respect to the targeted error $\delta^{*}$. Computations are conducted for $L=0.1$. Curves are generated for different $D$, as indicated.

shown in Table 1, the KL decomposition problem is recast in a reduced one that has a dimension ranging from $n_{t}=431$ to 3,840 , indicating a significant complexity reduction compared to the direct approach. For example, using intermediate value $D=160$, the DD-KL approach involves the solution of 160 independent eigenproblems (possibly in parallel) with size roughly $40,802 / 160=255$, and the resolution of the reduced problem with size $n_{t}=983$; these have to be compared with the direct approach in which one has to solve a single eigenproblem with dimension 40,802.

To complete the discussion, we provide in Figure 8 an analysis of the dependence of $n_{t}$ on the requested precision $\delta^{*}$ for different numbers of subdomains. The results reported in Figure 8 show the increase of $n_{t}$ with both $D$, as previously discussed, and the requested precision $\delta^{*}$, as one would have expected. However, it is seen that $n_{t}$ tends to level off when $\delta^{*}$ becomes very small. This trend can be again explained by the convergence of the local spectra. Note also the wavy behavior at low precision (largest $\delta^{*}$ ) and for the largest $D$ tested, which can be explained by the relative variability of the local basis dimension from a subdomain to another for these cases.

It should be clear at this point that the efficiency of the DD-KL method depends on the behavior of the local problems when the domain is partitioned into smaller ones. It is then important to investigate the impact of the partitioning method on this behavior. There are several approaches available to decompose $\Omega$ into $D$ nonoverlapping subdomains $\Omega_{d}$. We briefly mention two popular families: graph partitioning and $k$-means clustering. The graph partitioning methods (see, e.g., [13]) convert the mesh into a dual graph, in which the vertices are the mesh elements and edges link neighboring elements. The graph is then partitioned so as to balance the number of vertices in each partition, while minimizing the number of edges that straddle different partitions. The $k$-means clustering $[18,8,9,16]$ consists in partitioning data points into $k$ clusters, where each data point is assigned to the cluster whose mean (or centroid) is the nearest. In the context of domain decomposition, the data points to be partitioned correspond to the mesh element centroids or nodes. In this section, we rely on the $k$-means clustering method and consider different distance functions to control the geometrical properties of the subdomains $\Omega_{d}$. Specifically, we consider an anisotropic Euclidean distance,

$$
\Delta_{d}\left(\boldsymbol{x}, \boldsymbol{x}^{\prime}\right)=\sqrt{\left(x-x^{\prime}\right)^{2}+\rho\left(y-y^{\prime}\right)^{2}},
$$

to control with $\rho>0$ the aspect-ratio of the $\Omega_{d}$ and with $R>1$ control the dispersion of the subdomain sizes by using coefficients $r_{d}$, drawn uniformly in $[1, R]$, to scale the distance to the $d$ th centroid. Figure 9 shows several partitions of $\Omega$ into $D=20$ 
subdomains using different values of $\rho$ and $R$. It is seen that as $\rho>1$ increases (from left to right) the subdomains are more and more stretched horizontally. Further, for $R=1$ (top row) the subdomains have all similar size (surface), in contrast to the case with $R=10$ (bottom row) where a significant dispersion of the subdomains size is seen.

Before investigating the effects of the partition on the DD-KL method, we first check in Figure 10 that changing the geometry of the subdomains does not affect the accuracy of the method and that the selection of the local and global truncations remains appropriate. The plots confirm that, for all partitions tested, the method achieves the desired accuracy set with $\delta^{2}=2 \times 10^{-3}$.

Plotted in Figure 11 are the evolutions of the reduced problem size, $n_{t}$, as a function of the anisotropy parameter $\rho$ for different values of $R$ and $D$. Consistently with the results reported in Table $1, n_{t}$ increases with $D$ when $R$ and $\rho$ are held fixed. In addition, an effect related to the aspect-ratio of the subdomains is evidenced in Figure 11. Specifically, the size of the reduced problem is adversely affected for stretched subdomains. This effect was anticipated from the trends and behaviors reported above: the most effective reduction of the local bases is expected for subdomains that have well-balanced size in all directions. Incidentally, this behavior means that, in the case of anisotropic covariance functions, one would have interest in designing a partition of $\Omega$ that fits with the principal directions of $C$. We remark, however, that the effect of stretched subdomains is not so severe, with a reported increase of less than $50 \%$ in the reduced problem size when going from 1:1 to 1:20 aspect-ratios. Note

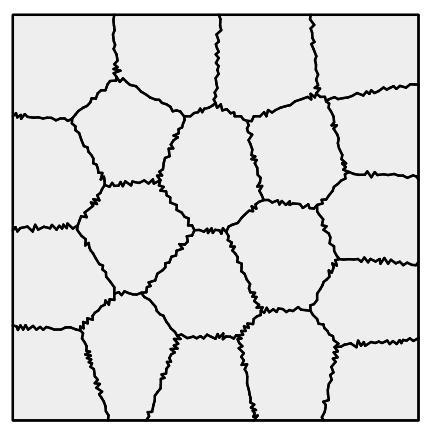

(a) $\rho=1, R=1$

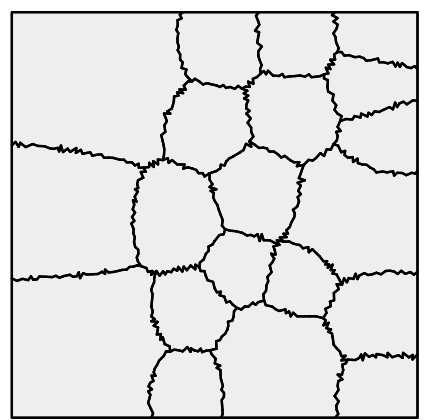

(d) $\rho=1, R=10$

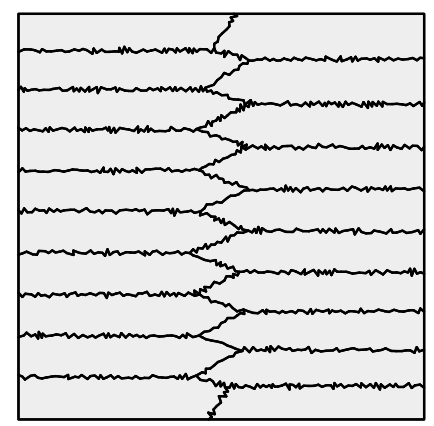

(b) $\rho=5, R=1$

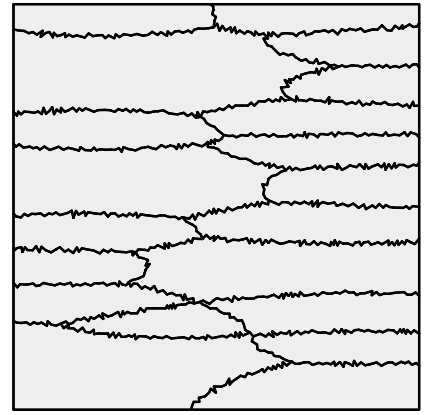

(e) $\rho=5, R=10$

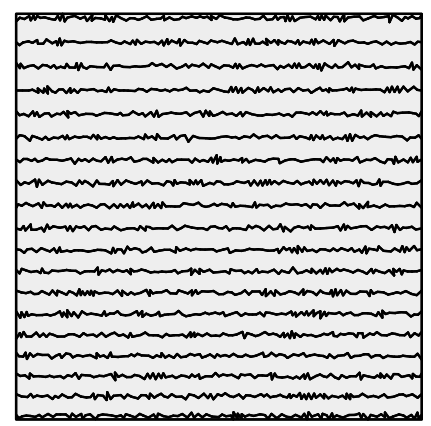

(c) $\rho=20, R=1$

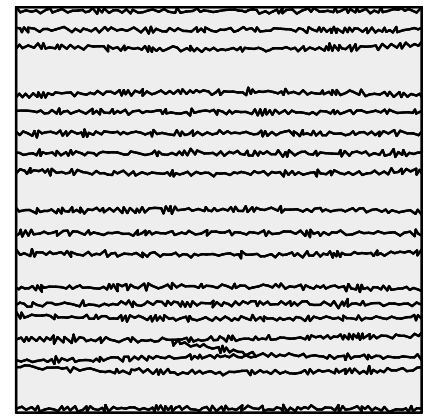

(f) $\rho=20, R=10$

FIG. 9. Partitioning of $\Omega=(0,1)^{2}$ into $D=20$ subdomains with the $k$-means clustering method, using different parameter values of $\rho$ and $R$ as indicated. 


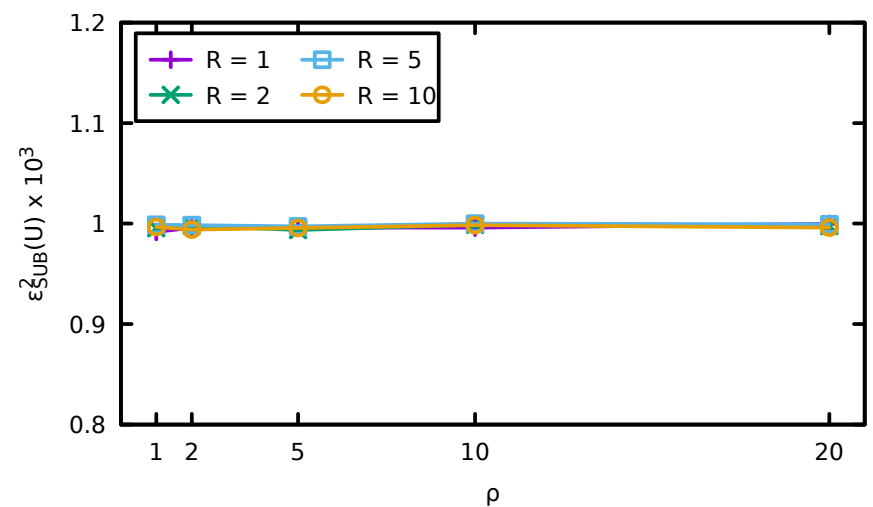

FIG. 10. $\epsilon_{\text {sub }}^{2}(U)$ versus $\rho$ for a target accuracy $\delta^{2}=2 \times 10^{-3}$. The domain is partitioned into $D=80$ subdomains using the $k$-means clustering with parameters $\rho$ and $R$ as indicated.

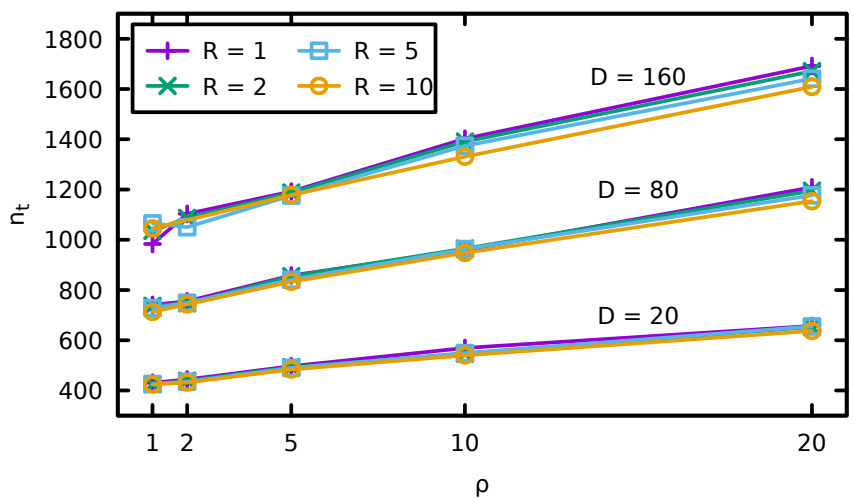

FIG. 11. $n_{t}$ versus $\rho$ for a target accuracy $\delta^{2}=2 \times 10^{-3}$. Curves are generated for different values of $D$ and $R$ as indicated.

that this robust behavior is not expected to depend on the correlation length of the process, provided that $D$ is large enough. This effect can be understood by recognizing that it is the ratio of correlation length to the characteristic size of the subdomain that governs the dimension of the local basis for a fixed stretching ratio. Finally, we observe from Figure 11 that increasing the dispersion of the subdomains size (increasing $R$ ) has a negligible effect on $n_{t}$. This can be explained by compensation effects between larger $m_{d}$ for large subdomains and lower $m_{d}$ for smaller ones. Having $n_{t}$ roughly independent of $R$ does not necessarily translate into a constant computational cost. In fact, having subdomains with very different sizes could induce severe load balancing issues for the parallel resolution of the local problems. On the positive side, being able to tune the geometric size of the subdomains, without impacting much $n_{t}$, means that one can eventually adapt the partition in the case of a nonuniform spatial discretization.

4. Performance analysis. This section is dedicated to assessing the serial behavior of our approach, as well as its parallel scalability and efficiency.

4.1. Serial behavior. We first investigate the influence of the number of subdomains $D$ on the computational time of the method in a serial implementation. We 
only report the behavior of the local solves (see (14)) and the assembly of the reduced problem, i.e., the filling of the block matrices (see (21)) because, for the range of $D$ values and the precision considered in the serial tests, the solution time for the reduced problem is dominated by the computational times of the first two steps. In addition, the variations in $n_{t}$ would have required selecting different eigensolvers (direct or iterative) for a fair comparison of the computational times for the reduced problem solution.

Figure 12 depicts the dependence of the computational times of these first two stages on the number of subdomains. We also compare the case of a discretization with either piecewise constant $\left(P_{0}\right)$ and piecewise quadratic $\left(P_{2}\right)$ finite elements in Figure 12(a) and (b), respectively. The computational times are reported in arbitrary units.

The first observation that can be drawn from this figure is that the two computational times decrease as the number of subdomains increases, with a faster decay for the local solves than the assembly of reduced problem, and the same behavior is reported for the two FE discretizations. Due to the faster decay of the local solves CPU time, the reduced problem assembly time becomes dominant as $D$ increases, and the combined time becomes quickly dominated by the matrix fill.

It is easy to understand that solving the local problems becomes cheaper as the number of subdomains increases: as $D$ increases, the subdomains become smaller, and thus the local problems involving fewer unknowns are cheaper to solve. In the tests presented, a direct solver was used for the local problem, so the computational time reduction is very significant. Another important advantage of the proposed approach stems from the independence of the local solves. While solving the global generalized eigenvalue problem (11) would require the evaluation and possibly the storage of the full stiffness matrix $[K]$ in (12), the local problems only involve stiffness matrices $\left[K^{(d)}\right]$ that correspond to pairs of points $\left(\boldsymbol{x}, \boldsymbol{x}^{\prime}\right)$ belonging to a same subdomain $\Omega_{d}$. In other words, the set of local matrices $\left[K^{(d)}\right]$ simply correspond to the diagonal, subdomain-based blocks of the global matrix $[K]$. This can result in significant CPU time and memory savings as compared to solving the global problem (11).

The reason for the reduction of the computational time for the reduced problem assembly is less obvious but can be explained as follows. A given block $\left[\hat{K}_{i, j}\right]$ has $m_{i} \times m_{j}$ entries, each one requiring an integration over $\Omega_{i} \times \Omega_{j}$, with complexity proportional to $\mathrm{Ne}_{i} \times \mathrm{Ne}_{j}\left(\mathrm{Ne}_{d}\right.$ being the number of degrees of freedom of the

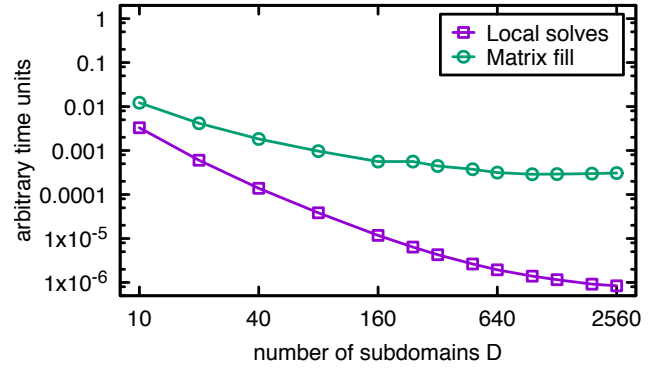

(a) $P_{0}$ finite elements.

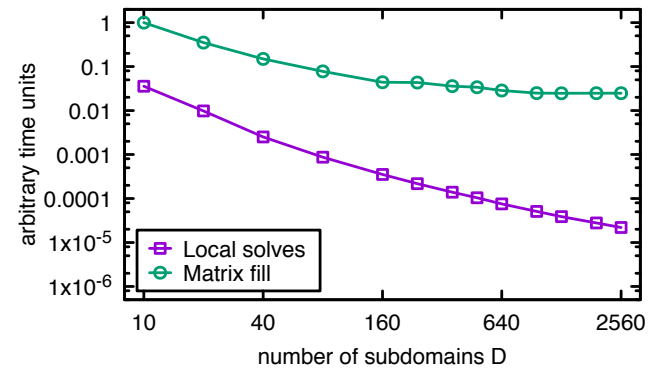

(b) $P_{2}$ finite elements.

FIG. 12. Computational times of local solves and reduced problem assembly versus D. Computations are performed on a fixed mesh with $\mathrm{Ne}=16,441$ dimensional finite element space. The correlation length is $L=0.1$, and the target accuracy is $\delta^{2}=10^{-3}$. 
approximations in $\Omega_{d}$ ). If the subdomains have roughly the same numbers of local modes $\left(m_{i} \approx m_{j} \approx \bar{m}\right)$ and unknowns $\left(\mathrm{Ne}_{i} \approx \mathrm{Ne}_{j} \approx \mathrm{Ne} / D\right)$, and given that there are $D(D+1) / 2$ such blocks to be computed (accounting for the symmetric structure), the complexity in assembling the reduced matrix $[\hat{K}]$ can be estimated to be proportional to $\mathrm{Ne}^{2} \bar{m}^{2} \times(D+1) / D$. In section 3 , we have seen that the average number of local modes $\bar{m}=n_{t} / D$ tends to decrease with $D$ (see Table 1 ), although $n_{t}$ increases. Consequently, the complexity of the reduced problem assembly reduces with $D$ though it is seen to progressively level off for very large number of subdomains. Eventually, as $m_{d}$ cannot be less than 1 , increasing further $D$ would have a negative impact on the overall efficiency, because though the assembling stage may not be penalized, the resolution cost of the reduced problem would continuously increase.

Finally, from Figure 12(a) and (b) it is found that the $P_{2}$ discretization is more expensive, in terms of computational times, regardless of the number of subdomains. This is due to a higher number of unknowns Ne for the $P_{2}$ discretization compared to the $P_{0}$ case when using the same triangulation of the computational domain. But a fair comparison between the two discretizations should account not only for the computional times but also for the spatial discretization error on the computed modes.

4.2. Parallel implementation. Our domain decomposition approach naturally lends itself to parallel computation. In addition to the reduced complexity of solving smaller problems, as reported above, further gain can be expected through parallelization. From the presentation of the method given in section 2 and summarized in Algorithm, one can identify the three main stages of the approach. First, local generalized eigenvalue problems are solved at the subdomain level (see (14)); second, the stiffness matrix of the reduced (regular) eigenvalue problem is constructed (see (21)); and last, this reduced problem is solved. We shall focus here on the first two stages, which represent the core of our approach. Regarding the last stage, let us simply mention that libraries exist to efficiently deal with the parallel solution of reduced problem, for example, PARPACK (Parallel ARPACK) [20], SLEPc (based on PETSc) [10, 24], or the Anasazi package of Trilinos [2].

In what follows, we rely on a message passing interface (MPI) approach to parallelize the solution of the local problems and the assembly of the reduced problem. We shall denote by $N_{\mathrm{MPI}}$ the number of MPI processes; we then split the set of subdomains into $N_{\text {MPI }}$ subsets. Each process $p$ holds the data corresponding to the $D_{p}$ subdomains in the $p$ th subset. In a static a priori load-balancing approach, provided that the number of degrees of freedom are roughly constant from one subdomain to another, we would like the number of subdomains $D_{p}$ handled by process $p$ to be evenly distributed among the processes, that is, $D_{p}=D / N_{\text {MPI }}$ for all $p$. In practice, to tackle the case where $N_{\mathrm{MPI}}$ does not divide $D$, we distribute the subdomains among the processes through

$$
\forall p=1, \ldots, N_{\mathrm{MPI}}, \quad D_{p}=\left\lfloor D / N_{\mathrm{MPI}}\right\rfloor+ \begin{cases}1 & \text { if } p \leq\left(D \bmod N_{\mathrm{MPI}}\right) \\ 0 & \text { otherwise }\end{cases}
$$

where $\lfloor\cdot\rfloor$ is the floor function. Such a distribution among processes is illustrated in Figure $13(\mathrm{a})$, in a case where $N_{\text {MPI }}$ does not divide $D$.

4.2.1. Parallel resolution of the local problems. In the first stage, the local problems are solved; they consist of the Fredholm equations defined by (14), which after discretization lead to the generalized eigenvalue problems (see Algorithm):

$$
\left[K^{(d)}\right]\left\{\tilde{\phi}_{\beta}^{(d)}\right\}=\lambda_{\beta}^{(d)}\left[M^{(d)}\right]\left\{\tilde{\phi}_{\beta}^{(d)}\right\} .
$$




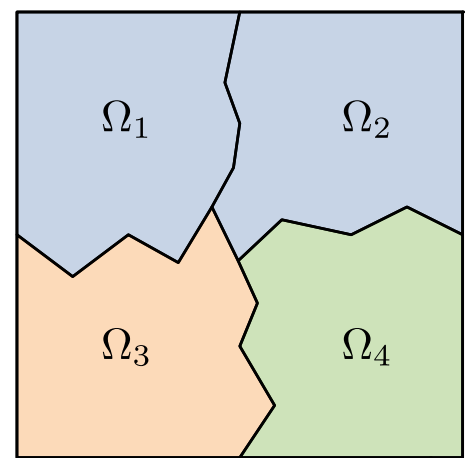

(a) Distribution of subdomains for the parallel resolution of the local problems.

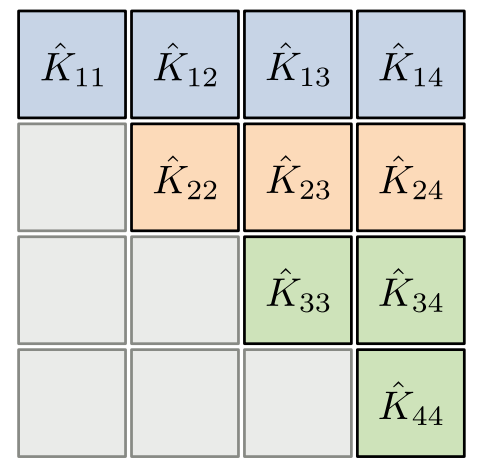

(b) Distribution of blocks for the parallel reduced problem assembly.

FIG. 13. Example of distribution among MPI processes of the subdomains (Figure 13(a)) and block computation for the reduced problem assembly (Figure 13(b)). Case of $D=4$ subdomains and $N_{\mathrm{MPI}}=3 \mathrm{MPI}$ processes. The processes $p=1,2,3$ are colored in blue (top line), orange (second line), and green (last two lines), respectively.

It is clear that these problems are independent for each subdomain and that only local data is needed to construct the stiffness and mass matrices $\left[K^{(d)}\right]$ and $\left[M^{(d)}\right]$. The parallel implementation of the local problems is thus trivial, as no communication is required between the corresponding MPI processes. Similarly, the local eigenvalues $\lambda_{\beta}^{(d)}$ and local eigenvectors $\tilde{\phi}_{\beta}^{(d)}$, for $\beta=1, \ldots, m_{d}$, can be conveniently stored locally in a distributed memory architecture. Because there are $D_{p}$ subdomains held by process $p$, and one single local eigenvalue problem to be solved per subdomain, process $p$ is thus in charge of solving $D_{p}$ problems. Provided that the number of degrees of freedom are roughly constant from one subdomain to another, we could expect the workload to be approximately balanced among the MPI processes and thus lead to good parallel performance.

4.2.2. Construction of the reduced eigenvalue problem. The second stage differs from the previous one in many ways. Perhaps the most relevant difference is the need to access nonlocal data. Indeed, as can be observed from (21), the blockmatrices $\left[\hat{K}_{i, j}\right]$ involve data that belong to subdomains $\Omega_{i}$ and $\Omega_{j}$. For diagonal blocks $(i=j)$, or for blocks corresponding to subdomains that are handled by the same process $p$, no MPI communication is required, as all the needed data is held by the same process. On the contrary, for other blocks, data have to be exchanged between different MPI processes, namely, the local modes $\tilde{\phi}_{\beta}^{(d)}$, as well as local mesh information for discretizing the integrals. This is handled by resorting to an all-to-all communication, specifically to the MPI function MPI_Allgatherv.

Another important difference lies in the fact that the total number of blocks, $N_{K} \equiv D^{2}$, grows quadratically with the number of subdomains $D$. The classical way of distributing the workload would be for each process $p$ to handle the construction of the $D_{p}$ block-rows corresponding to its subdomains. In other words, process $p$ would compute $\left[\hat{K}_{i, j}\right]$ for all $j=1, \ldots, D$ and for all $i \in \mathcal{I}_{p}$, where $\mathcal{I}_{p}$ denotes the set of subdomain indices handled by process $p$. Here, we further exploit the characteristic block-symmetric structure of the reduced problem matrix inherited from the symmetry of the covariance functions $C$. Specifically, we compute only the $N_{K}^{\triangle} \equiv D(D+1) / 2$ 
upper (or lower) triangular blocks of the matrix. The computation of these $N_{K}^{\triangle}$ blocks is distributed among the $N_{\text {MPI }}$ processes so that process $p$ is in charge of $N_{K, p}^{\triangle}$ blocks. The distribution of the blocks on the different processes is again handled using (43), substituting $D$ and $D_{p}$ with $N_{K}^{\triangle}$ and $N_{K, p}^{\triangle}$, respectively. The distribution among processes is illustrated in Figure 13(b), in a case where $N_{\text {MPI }}$ does not divide $N_{K}^{\triangle}$.

The cost of computing each block matrix $\left[\hat{K}_{i, j}\right]$ depends both on the number of degrees of freedom in subdomains $\Omega_{i}$ and $\Omega_{j}$ and on the number of local modes $m_{i}$ and $m_{j}$ retained for these subdomains. Provided that these are balanced, the workload of constructing the reduced problem should also be balanced among the processes and thus lead to good parallel performance. More general situations could be considered, in particular in the case of nonuniform mesh and nonisotropic covariance structure, by introducing more advanced load-balancing strategies. This will be addressed in follow-on work.

4.3. Parallel efficiency. We now turn to investigate the efficiency of the parallel implementations of the local problem solves and the assembly of the reduced problem matrix. Specifically, we measure the scalability with the number of processes of performing these two tasks in parallel, including the MPI communication times involved. The scalability is characterized using two quantities, the parallel speedup $S$ and the parallel efficiency $E$. The speedup and efficiency are reported as functions of the number $N_{\text {MPI }}$ of MPI processes considered. The two measures are defined by

$$
S\left(N_{\mathrm{MPI}}\right) \equiv T(1) / T\left(N_{\mathrm{MPI}}\right), \quad E\left(N_{\mathrm{MPI}}\right) \equiv 100 \times S\left(N_{\mathrm{MPI}}\right) / N_{\mathrm{MPI}},
$$

where $T\left(N_{\mathrm{MPI}}\right)$ is the measured CPU times for the execution of the tasks using $N_{\mathrm{MPI}}$ processes. As the smallest number of processes tested is $N_{\mathrm{MPI}}=16$, the definitions of speedup and efficiency are actually based on the approximation $T(1) \approx 16 T(16)$, assuming a perfect speedup for 16 processes. The tests were carried out on a parallel Blue Gene machine, fixing a constant number of MPI processes per computational node. For simplicity, we only report here the case with a single MPI process per node, such that the parallel runs use as many nodes as processes.

For a fixed finite element mesh with $\mathrm{Ne}=40,802$, we tested three different partitions of the domain, considering $D \in\{256 ; 512 ; 768\}$. A finer mesh with $\mathrm{Ne}=81,753$ degrees of freedom is also tested with $D=256$ subdomains. The problem size being kept constant as the number of processes is increased, the reported speedup and efficiency correspond to a strong scaling experiment.

The results are reported in Figure 14. Globally, the speedup and efficiency evolve satisfactorily as $N_{\mathrm{MPI}}$ is increased, especially considering that no fine-tuning of the parallel implementation has been performed. For the largest number of subdomains tested, $D=512$ and $D=768$, the parallel efficiency decreases slowly with $N_{\mathrm{MPI}}$, down to approximately $85 \%$ for 256 processes. This is due to the fact that the local problems are relatively small, owing to the large number of subdomains, making the MPI communication time more significant compared to the actual computation time. This trend becomes more pronounced as $N_{\mathrm{MPI}}$ increases.

For $D=256$, the actual computational time is large enough compared to the communication time, even for $N_{\mathrm{MPI}}=256$ processes. Consequently, the parallel efficiency remains close to ideal when $N_{\text {MPI }}$ increases, with a reported efficiency of about $97 \%$ with 256 processes. This effect is confirmed considering a finer finite mesh with $\mathrm{Ne}=81,753$ elements for the same $D(256)$. In this case, a higher efficiency of nearly $99 \%$ is measured for $N_{\mathrm{MPI}}=256$. 

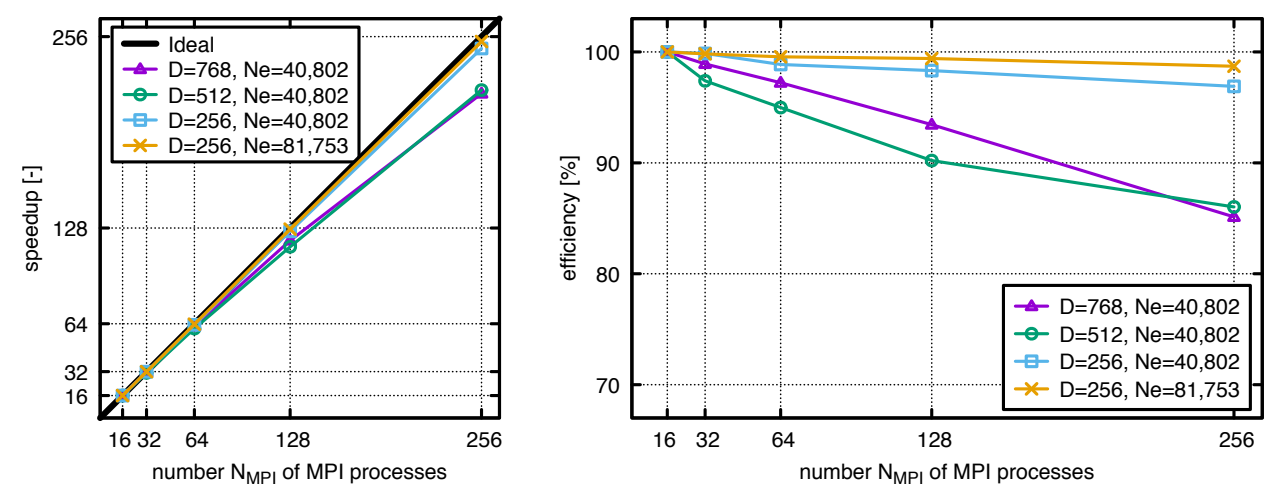

FIG. 14. Speedup (left) and efficiency (right) versus the number of MPI processes. Plotted are curves for different values of $D$ and $\mathrm{Ne}$ as indicated. The ideal scaling law is shown on the left plot using a solid black line. The correlation length is $L=0.1$, and the energy criterion for the local modes is $\delta^{2} / 2=10^{-3}$.

These tests demonstrate that the DD-KL approach is efficient and scalable. Not only does it benefit from cost reduction owing to the domain decomposition that breaks the problem in a set of small independent subproblems, but it also lends itself to natural parallel processing, involving limited communication, and thus enabling the efficient computation of the KL decomposition for large problems.

5. Conclusions. In this paper, we presented an efficient method to solve largescale KL decomposition problems, based on a work-subdivision strategy. Specifically, the computational domain is partitioned into smaller nonoverlapping subdomains, over which local KL decomposition problems are solved to generate local bases. A criterion has been proposed, and numerically demonstrated, for the selection of the local bases' dimensions in order to ensure a prescribed accuracy. The global KL decomposition problem is subsequently reformulated by means of Galerkin projection in the subspace of the local modes. This procedure leads us to solve a reduced global problem, whose size is not related to the dimension of the underlying discretization space but depends on the requested accuracy and the number of subdomains. The low dimensionality of the reduced problem enables efficient solution methods, including direct ones. But the approach is in fact flexible and can accommodate any type of eigenvalue solvers (direct, iterative, etc.), both for the computing the local KL bases and solving of the reduced problem. In addition, although we focused on the problem of performing KL decomposition, it should be noted that the approach could be used in many different contexts and extended to determine the dominant subspace associated to more general integral operators.

The method was illustrated on the approximation of stochastic processes defined over the two-dimensional unit square. A squared exponential covariance structure was assumed, with short correlation lengths yielding slowly decaying spectra. Our numerical experiments demonstrated that the approach provides a fine control of the approximation error and is very robust with respect to the number of subdomains. It was also observed that the cost of solving the local problems reduces with the number $D$ of subdomains, while the size and cost of solving the reduced problem increases with $D$. This suggests the existence of an optimal value for $D$, balancing the decreasing complexity of the local KL problems and the increasing size of the reduced problem. In any case, the range of values of $D$ over which the approach remains 
effective is large such that selecting the optimal $D$ is not critical. The sensitivity of the method to the shape of the subdomains was also explored. It was found that, in the case of isotropic covariance, it is more effective to use partitions with subdomains having similar size in all directions, rather than having geometrically highly stretched subdomains. In any case, the influence of the subdomains' geometry is not too pronounced, such that the method would be able to effectively accommodate complicated situations (in the covariance structure, geometry of the domain, adapted finite element mesh, etc.) without having to construct a dedicated partitioner. This is particularly important in view of reusing standard libraries for partitioning the domain.

Beside the immediate computational advantages of breaking the scale size problem into smaller ones, the proposed approach naturally lends itself to parallel implementation. In fact, the parallelization of the local decompositions over individual subdomains is trivial, as local problems are fully independent from one subdomain to another, and the error criterion to select the local basis is also completely local. The parallel assembly of the reduced problem involves, on the contrary, more significant communication between processes, with a less obvious parallelization as a result. The parallel implementation, relying on the MPI framework, has been tested showing an excellent scaling up to 256 processes, provided that the initial global problem is large enough.

The proposed approach allows us to effectively generate approximated samples of a Gaussian process, using (27). Thus, as discussed in the introduction, the method is therefore an alternative to other existing methods to generate realizations of Gaussian stochastic processes with prescribed covariance structure. In fact, the solution of the reduced problem exhibits the correlation structure between local variables, such that one can also restrict the sampling to subsets of subdomains. Such a sampler is advantageous, for instance, to solve stochastic PDE problems, in particular stochastic elliptic problems as considered in the second part of the paper.

Appendix A. Derivation of the error expression. We have an initial process $U(\boldsymbol{x}, \theta) \in L_{2}(\Omega, \Theta)$, its projection $U_{\mathscr{B}}(\boldsymbol{x}, \theta) \in \mathcal{V}_{\mathscr{B}} \times L_{2}(\Theta)$, and finally $\hat{U}(\boldsymbol{x}, \theta) \in$ $\mathcal{V}_{\mathscr{B}} \times L_{2}(\Theta)$, the truncated KL expansion of $U_{\mathscr{B}}$ using $\hat{N} \leq n_{t}$ modes. Our concern is to derive an expression for the error $U-\hat{U}$ in the $L_{2}(\Omega, \Theta)$-norm. By definition this is the square root of

$$
\mathbb{E}\left[\|U-\hat{U}\|_{\Omega}^{2}\right]=\mathbb{E}\left[\langle U-\hat{U}, U-\hat{U}\rangle_{\Omega}\right] .
$$

We have

$$
\begin{aligned}
\langle U-\hat{U}, U-\hat{U}\rangle_{\Omega}= & \left\langle\left(U-U_{\mathscr{B}}\right)+\left(U_{\mathscr{B}}-\hat{U}\right),\left(U-U_{\mathscr{B}}\right)+\left(U_{\mathscr{B}}-\hat{U}\right)\right\rangle_{\Omega} \\
= & \left\langle\left(U-U_{\mathscr{B}}\right),\left(U-U_{\mathscr{B}}\right)+\left(U_{\mathscr{B}}-\hat{U}\right)\right\rangle_{\Omega} \\
& \quad+\left\langle\left(U_{\mathscr{B}}-\hat{U}\right),\left(U-U_{\mathscr{B}}\right)+\left(U_{\mathscr{B}}-\hat{U}\right)\right\rangle_{\Omega} \\
= & \left\|U-U_{\mathscr{B}}\right\|_{\Omega}^{2}+\left\|U_{\mathscr{B}}-\hat{U}\right\|_{\Omega}^{2}+2\left\langle\left(U-U_{\mathscr{B}}\right),\left(U_{\mathscr{B}}-\hat{U}\right)\right\rangle_{\Omega} .
\end{aligned}
$$

However, the last term is zero as $\left(U_{\mathscr{B}}-\hat{U}\right) \in \mathcal{V}_{\mathscr{B}} \times L_{2}(\Theta)$ while $\left(U-U_{\mathscr{B}}\right)$ is orthogonal to $\mathcal{V}_{\mathscr{B}} \times L_{2}(\Theta)$. Then it follows that

$$
\mathbb{E}\left[\|U-\hat{U}\|_{\Omega}^{2}\right]=\mathbb{E}\left[\left\|U-U_{\mathscr{B}}\right\|_{\Omega}^{2}+\left\|U_{\mathscr{B}}-\hat{U}\right\|_{\Omega}^{2}\right]=\mathbb{E}\left[\left\|U-U_{\mathscr{B}}\right\|_{\Omega}^{2}\right]+\mathbb{E}\left[\left\|U_{\mathscr{B}}-\hat{U}\right\|_{\Omega}^{2}\right] .
$$


Acknowledgments. The parallel experiments were carried out on the IBM Blue Gene/Q machine of IDRIS (CNRS, Orsay), and the authors would like to thank J. Chergui for helpful discussions and his valuable assistance in carrying out the parallel experiments.

\section{REFERENCES}

[1] K. E. Atkinson, The Numerical Solution of Integral Equations of the Second Kind, Cambridge University Press, Cambridge, 1997.

[2] C. G. Baker, U. L. Hetmaniuk, R. B. Lehoucq, and H. K. Thornquist, Anasazi software for the numerical solution of large-scale eigenvalue problems, ACM Trans. Math. Software, 36 (2009), 13.

[3] W. Betz, I. Papaioannou, and D. Straub, Numerical methods for the discretization of random fields by means of the Karhunen-Loève expansion, Comput. Methods Appl. Mech. Engrg., 271 (2014), pp. 109-129.

[4] O. Ditlevsen and N. TARP-Johansen, Choice of input fields in stochastic finite-elements, Probabilist. Eng. Mech., 14 (1999), pp. 63-72.

[5] R. G. Ghanem and P. D. Spanos, Stochastic Finite Elements: a Spectral Approach, Courier Corporation, Chelmsford, MD, 2003.

[6] G. Golub and C. Van Loan, Matrix Computations, Johns Hopkins University Press, 3rd ed., Baltimore, MD, 1996.

[7] R. Gutiérrez, J. C. Ruiz, and M. J. Valderrama, On the numerical expansion of a second order stochastic process, Appl. Stoch. Models Bus. Ind., 8 (1992), pp. 67-77.

[8] J. A. Hartigan, Clustering Algorithms, John Wiley \& Sons, New York, 1975.

[9] J. A. Hartigan and M. A. Wong, Algorithm AS 136: A k-means clustering algorithm, J. R. Stat. Soc. Ser. C. Appl. Stat., 28 (1979), pp. 100-108.

[10] V. Hernandez, J. E. Roman, and V. Vidal, SLEPc: A scalable and flexible toolkit for the solution of eigenvalue problems, ACM Trans. Math. Software, 31 (2005), pp. 351-362.

[11] M. Kac And A. Siegert, An explicit representation of a stationary Gaussian process, Ann. Math. Stat., 18 (1947), pp. 438-442.

[12] K. Karhunen, Uber lineare methoden in der wahrscheinlichkeitsrechnung, Ann. Acad. Sci. Fenn. Math. 37 (1947), pp. 3-79.

[13] G. KarYPIS AND V. KUmar, A fast and high quality multilevel scheme for partitioning irregular graphs, SIAM J. Sci. Comput., 20 (1998), pp. 359-392.

[14] O. Le Maître, M. Reagan, H. Najm, R. Ghanem, and O. Knio, A stochastic projection method for fluid flow. II. Random process, J. Comput. Phys. 181 (2002), pp. 9-44.

[15] O. P. Le Maître and O. M. KNiO, Spectral Methods for Uncertainty Quantification: With Applications to Computational Fluid Dynamics, Springer, New York, 2010.

[16] S. LlOYD, Least squares quantization in PCM, IEEE Trans. Inform. Theory, 28 (1982), pp. 129-137.

[17] M. LoÈve, Fonctions aléatoires du second ordre, in Processus Stochastique et mouvement Brownien, P. Lévy, ed. Gauthier-Villars, Paris, 1948.

[18] J. MACQUEEN, Some methods for classification and analysis of multivariate observations, in Proceedings of the Fifth Berkeley Symposium on Mathematical Statistics and Probability, Volume 1: Statistics, University of California Press, Berkeley, CA, 1967, pp. 281-297.

[19] Y. M. Marzouk AND A. F. Ghoniem, K-means clustering for optimal partitioning and dynamic load balancing of parallel hierarchical n-body simulations, J. Comput. Phys., 207 (2005), pp. $493-528$.

[20] K. J. Maschhoff and D. C. Sorensen, P_ARPACK: An efficient portable large scale eigenvalue package for distributed memory parallel architectures, in Applied Parallel Computing Industrial Computation and Optimization, J. Wásniewski, J. Dongarra, K. Madsen, and D. Olesen, eds. Springer, New York, 1996, pp. 478-486.

[21] H. G. Matthies, C. E. Brenner, C. G. Bucher, and C. G. Soares, Uncertainties in probabilistic numerical analysis of structures and solids-stochastic finite elements, Struct. Safety, 19 (1997), pp. 283-336.

[22] A. NouY, A generalized spectral decomposition technique to solve a class of linear stochastic partial differential equations, Comput. Methods Appl. Mech. Engrg., 196 (2007), pp. 4521-4537.

[23] K. Phoon, S. Huang, AND S. QueK, Implementation of Karhunen-Loève expansion for simulation using a wavelet-Galerkin scheme, Probabilist. Eng. Mech., 17 (2002), pp. 293-303.

Copyright $@$ by SIAM. Unauthorized reproduction of this article is prohibited. 
[24] J. E. Roman, C. Campos, E. Romero, and A. Tomas, SLEPc Users Manual, Technical Report DSIC-II/24/02 - Revision 3.6, Departamento de Sistemas Informáticos y Computación, Universitat Politècnica de València, 2015.

[25] C. Schwab AND R. A. Todor, Karhunen-Loève approximation of random fields by generalized fast multipole methods, J. Comput. Phys., 217 (2006), pp. 100-122.

[26] G. Stefanou, The stochastic finite element method: Past, present and future, Comput. Methods Appl. Mech. Engrg., 198 (2009), pp. 1031-1051.

[27] B. Sudret And A. Der Kiureghian, Stochastic Finite Element Methods and Reliability: A State-of-the-Art Report, Department of Civil and Environmental Engineering, University of California, Berkeley, CA, 2000.

[28] L. Tamellini, O. Le Maître, and A. Nouy, Model reduction based on proper generalized decomposition for stochastic steady incompressible Navier-Stokes equations, SIAM J. Sci. Comput., 36 (2014), pp. A1089-A1117.

Copyright (c) by SIAM. Unauthorized reproduction of this article is prohibited. 